\title{
Autoantibodies targeting TRIM72 compromise membrane repair and contribute to inflammatory myopathy
}

\author{
Kevin E. McElhanon, ${ }^{1}$ Nicholas Young, ${ }^{2}$ Jeffrey Hampton, ${ }^{2}$ Brian J. Paleo, ${ }^{1}$ Thomas A. Kwiatkowski, ${ }^{1}$ Eric X Beck, ${ }^{1}$ Ana Capati, ${ }^{1}$ \\ Kyle Jablonski, ${ }^{2}$ Travis Gurney, ${ }^{1}$ Miguel A. Lopez Perez, ${ }^{1}$ Rohit Aggarwal, ${ }^{3}$ Chester V. Oddis, ${ }^{3}$ Wael N. Jarjour, ${ }^{2}$ and Noah Weisleder ${ }^{1}$ \\ 'Dorothy M. Davis Heart and Lung Research Institute and Department of Physiology and Cell Biology, and 'Division of Rheumatology and Immunology, Department of Internal Medicine, College of Medicine, \\ The Ohio State University Wexner Medical Center, Columbus, Ohio, USA. ${ }^{3}$ Division of Rheumatology and Clinical Immunology, University of Pittsburgh School of Medicine, Pittsburgh, Pennsylvania, USA.
}

\begin{abstract}
Idiopathic inflammatory myopathies (IIM) involve chronic inflammation of skeletal muscle and subsequent muscle degeneration due to an uncontrolled autoimmune response; however, the mechanisms leading to pathogenesis are not well understood. A compromised sarcolemmal repair process could promote an aberrant exposure of intramuscular antigens with the subsequent initiation of an inflammatory response that contributes to IIM. Using an adoptive transfer mouse model of IIM, we show that sarcolemmal repair is significantly compromised in distal skeletal muscle in the absence of inflammation. We identified autoantibodies against TRIM72 (also known as MG53), a muscle-enriched membrane repair protein, in IIM patient sera and in our mouse model of IIM by ELISA. We found that patient sera with elevated levels of TRIM72 autoantibodies suppress sarcolemmal resealing in healthy skeletal muscle, and depletion of TRIM72 antibodies from these same serum samples rescues sarcolemmal repair capacity. Autoantibodies targeting TRIM72 lead to skeletal muscle fibers with compromised membrane barrier function, providing a continuous source of autoantigens to promote autoimmunity and further amplifying humoral responses. These findings reveal a potential pathogenic mechanism that acts as a feedback loop contributing to the progression of IIM.
\end{abstract}

\section{Introduction}

The idiopathic inflammatory myopathies (IIM) are a group of disorders characterized by a chronic state of inflammation leading to a degeneration of skeletal muscle structure and function (1-4). IIM (also known as myositis) includes several major clinical subtypes: polymyositis (PM), juvenile and adult dermatomyositis (JDM/ $\mathrm{DM})$, statin-induced necrotizing myopathy (SINM), and inclusion body myositis (IBM). Each subtype of IIM shares skeletal muscle inflammation as a common feature, though it can be mild or nonexistent in SINM. Clinically, muscle weakness can range from asymptomatic to severe, primarily in the proximal muscles. IIM affects adults with a wide age spectrum, while JDM primarily affects children starting at 2 years of age (5).

The histopathological features of PM include perivascular and endomysial inflammation consisting mainly of macrophages and $\mathrm{CD}^{+} \mathrm{T}$ cells, necrotic and regenerating muscle fibers, and variability in skeletal muscle fiber size (6). While the mechanism responsible for the pathogenesis of IIM remains largely unknown, $\mathrm{T}$ cell-mediated autoimmune responses contribute to the inflammation caused by both genetic and environ-

Authorship note: WNJ and NW are co-senior authors. Conflict of interest: NW is a founder of TRIM-edicine Inc. Copyright: () 2020, American Society for Clinical Investigation. Submitted: July 15, 2019; Accepted: May 14, 2020; Published: July 20, 2020. Reference information: J Clin Invest. 2020;130(8):4440-4455. https://doi.org/10.1172/JCI131721 mental factors (7-9). Two mouse models of IIM have provided new insights into the pathogenesis of IIM. The synaptotagmin 7-null $\left(S y t 7^{-/-}\right)$mouse is characterized by defects in sarcolemmal repair and develops a mild form of myositis at approximately 4 weeks of age with the resolution of inflammation over time (1012). The synaptotagmin family of proteins is known to facilitate the trafficking of vesicles to the site of an injury, forming a repair patch in the membrane resealing process (13-17). The development of IIM may be triggered by increased exposure of intramuscular antigens due to the defective membrane repair response (10). A more robust model of IIM combines knockout of Syt7 with a mutation in the Foxp3 gene, resulting in a mouse model with impaired membrane resealing capacity and a deficiency in $\mathrm{T}$ regulatory cells (Tregs) (18). Adoptive transfer of lymph node cell preparations isolated from $\mathrm{Foxp}^{3^{-/ Y}} \mathrm{Syt7^{-/ }}$ doublemutant mice into immunodeficient recombination-activating gene 1-null $\left(\mathrm{Ragl}^{-/}\right)$recipient mice results in severe inflammation, demonstrating that aberrant muscle antigen exposure due to defects in membrane resealing contributes to the onset and progression of IIM (12).

Plasma membrane repair is a highly conserved mechanism restoring the barrier function of the membrane after injury and is a critical component of maintaining normal cellular physiology (19-23). While a simple lipid bilayer will reseal through thermodynamic principles, the eukaryotic cytoskeleton necessitates that a lipid bilayer bordering the cell be held under a degree of mechanical tension. When held under tension, even small disruptions of 
a lipid bilayer cannot spontaneously reseal (24). Previous studies established the framework of the cell membrane repair response $(23,25,26)$. This process requires the translocation of intracellular vesicles to the injury site through the action of kinesin and myosin motor proteins (26). Vesicles fuse with the plasma membrane in a calcium-dependent manner to form a repair patch in a process similar to the release of neurotransmitters from neurons $(25,27)$. Disruption of the resealing process contributes to many diseases, including muscular dystrophy, heart failure, and neurodegeneration (28-30). Given the importance of the plasma membrane barrier function in preventing exposure of intracellular antigens to the extracellular space where they can act as autoantigens, it is plausible that compromised sarcolemmal resealing could contribute to IIM pathogenesis and progression.

Previous studies have linked the tripartite motif (TRIM) family of E3 ubiquitin ligase proteins to membrane repair and IIM. Several TRIM family proteins (Ro52, TIF1 $\alpha$, TIF1 $\beta$, and TIF1 $\gamma$ ) are known autoantigens associated with IIM (31-33), and the function of additional TRIM proteins in innate immunity continues to be discovered (34-36). Additionally, TRIM72 (also known as MG53) mediates sarcolemmal membrane repair in striated muscle (3739). The sarcolemmal membrane repair response is initiated by the influx of extracellular calcium at the site of injury (27). This triggers intracellular vesicle trafficking to the sarcolemma, forming a patch that allows the injured muscle fiber to survive (20, 21, $37,40)$. Previous studies from our group and others have shown that TRIM72 is a critical component of the sarcolemmal repair process in striated muscle $(37,38,41)$. Trim $72^{-/-}$mice develop significant skeletal muscle myopathy and cardiovascular defects due to defective sarcolemmal repair (42-44). Given the possible contribution of membrane repair in the development of IIM and the critical function of TRIM72 in the repair process, we hypothesized that immunological exposure to extracellular TRIM72 during the membrane repair response results in TRIM72 autoantibody production that can negatively affect membrane resealing and exacerbate autoimmune-mediated inflammation.

In the current study, we sought to identify TRIM family proteins acting as autoantigens in IIM and elucidate a potential mechanistic role of the resulting autoantibodies in disease progression. Our findings represent a mechanism that drives the progression of IIM when decreased sarcolemmal integrity, resulting from autoantibodies targeting a critical component of sarcolemmal resealing, induces a positive-feedback loop of muscle damage and aberrant intramuscular antigen presentation that directly contributes to the pathophysiology of IIM.

\section{Results}

Adoptive transfer of lymph node cells from Foxp $3^{-/ Y}$ Syt $7^{-1-}$ mice results in severe proximal muscle inflammation while sparing distal muscle. We have previously reported that the adoptive transfer of lymph node cell preparations from Foxp $3^{-/ Y} S y t 7^{-/-}$mice to $R a g 1^{-/-}$recipients results in severe inflammation of the quadriceps, with the inflammatory infiltrate consisting predominantly of $\mathrm{CD} 4^{+}$and $\mathrm{CD} 8^{+} \mathrm{T}$ cells, and a smaller number of macrophages (12). To determine whether our adoptive transfer model of myositis recapitulated the predominantly proximal muscle pattern of inflammation observed in IIM subjects, we performed H\&E staining on the quadriceps (proximal), extensor digitorum longus (EDL; distal), and soleus (distal) muscles of $\mathrm{Rag1}^{-/-}$recipient mice adoptively transferred with lymph node cell preparations from Foxp $3^{-/ Y} S y t 7^{-/-}$ mice and $R a g 1^{-/}$mice receiving sham adoptive transfer. Quadriceps muscles from mice receiving sham adoptive transfer were histologically normal at 1 week and 4 weeks after adoptive transfer with no inflammatory infiltrates or fatty tissue infiltrates observed (Figure 1A). Rag1-/- mice receiving adoptive transfer of lymph node cells from Foxp $3^{-/ Y}$ Syt $7^{-/-}$mice exhibited severe inflammation and fatty tissue infiltration in the quadriceps at 1 and 4 weeks after adoptive transfer (Figure 1A), which included both perivascular and endomysial inflammation.

$\mathrm{H} \& \mathrm{E}$ staining of the EDL from $\mathrm{Rag1}^{1 /-}$ mice receiving adoptive transfer of Foxp $3^{-/ Y} S y t 7^{-/}$lymph node cells showed little or no sign of inflammation, fatty tissue infiltration, or regenerating muscle fibers (Figure 1B). These findings demonstrate that our adoptive transfer model of myositis reproduces the proximal muscle pattern of inflammation observed in IIM subjects. Importantly, no skeletal muscle pathology was observed in the distal muscles examined (EDL, Figure 1B; and soleus, Supplemental Figure 1; supplemental material available online with this article; https://doi.org/10.1172/ JCI131721DS1), consistent with the characteristic proximal muscle distribution of inflammation observed clinically in IIM subjects.

Sarcolemmal integrity of distal skeletal muscle is compromised in the absence of inflammation. We hypothesized that decreased sarcolemmal integrity and/or sarcolemmal resealing capacity in muscle could lead to antigen exposure and drive IIM progression. To assess sarcolemmal integrity, we quantified the $\operatorname{IgG}^{+}$muscle fibers present in quadriceps, EDL, and soleus muscles isolated from C57BL mice and Rag1 ${ }^{-/-}$mice with adoptively transferred lymph node cells from Foxp $3^{-/ Y} S y t 7^{-/-}$mice at 1 week and 4 weeks. C57BL mice were chosen as controls owing to the lack of mature B

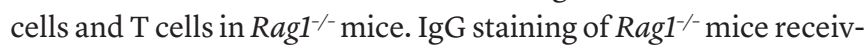
ing sham adoptive transfer was performed on EDL muscles to determine the nonspecific background and was found to be comparable to C57BL skeletal muscle (Supplemental Figure 2). Quadriceps from mice adoptively transferred with lymph node cells from Foxp3 $3^{-/ Y}$ Syt $7^{-/-}$mice showed a trend of decreased sarcolemmal integrity compared with C57BL controls at 1 week after adoptive transfer (Figure 2, A and C). Quadriceps from mice 4 weeks after adoptive transfer showed a significant decrease in sarcolemmal integrity (Figure 2, A and C). Surprisingly, the extent of $\operatorname{IgG}^{+}$ muscle fibers present in the EDL of mice receiving adoptive transfer of lymph node cells from Foxp $3^{-/ Y} S y t 7^{-/}$mice significantly increased at 1 and 4 weeks (Figure 2, B and D) and was greater than that observed in the quadriceps of mice 1 and 4 weeks after adoptive transfer. Decreased sarcolemmal integrity was also observed in soleus muscles from $\mathrm{Ragl}^{-/-}$mice receiving adoptive transfer of lymph node cells from Foxp $3^{-/ Y} S y t 7^{-/}$mice (Supplemental Figure 3). We did not observe any inflammation in EDL or soleus muscles stained with $\mathrm{H} \& \mathrm{E}$ (Figure 1B and Supplemental Figure 1); thus, the decrease in sarcolemmal integrity observed in distal muscle is not the result of direct immune cell-mediated damage.

Lack of Tregs alone does not induce a defect in sarcolemmal resealing. To determine whether an unchecked immune response is sufficient to induce sarcolemmal resealing defects, laser injury assays were performed on C57BL, Foxp $3^{-/ Y}$, and $S y t 7^{-/-}$mice. 
A

$1 \mathrm{wk}$

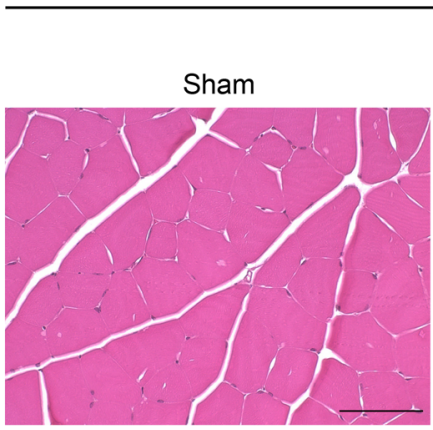

wk

Foxp3 $3^{-\wedge}$ Syt $^{-1-}$

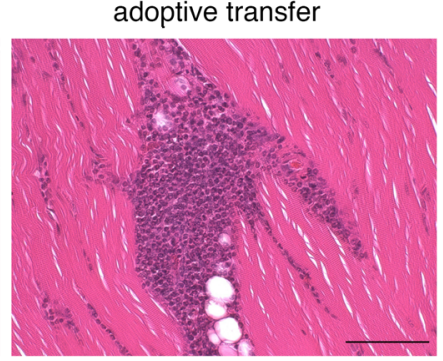

$4 \mathrm{wk}$

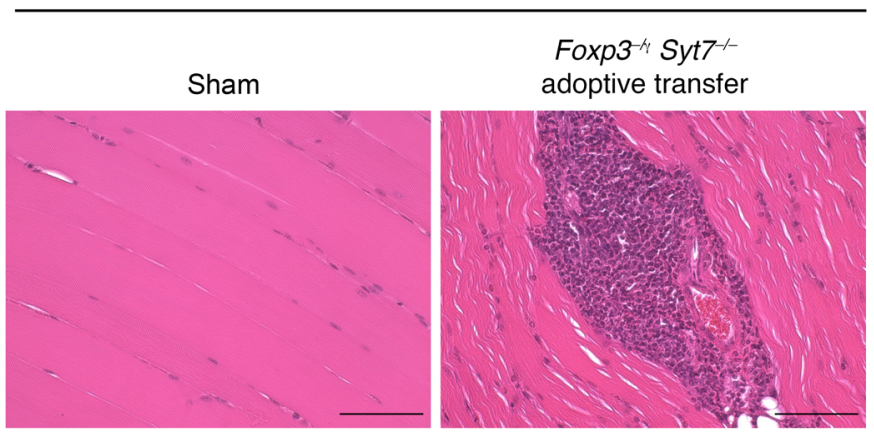

EDL

$1 \mathrm{wk}$

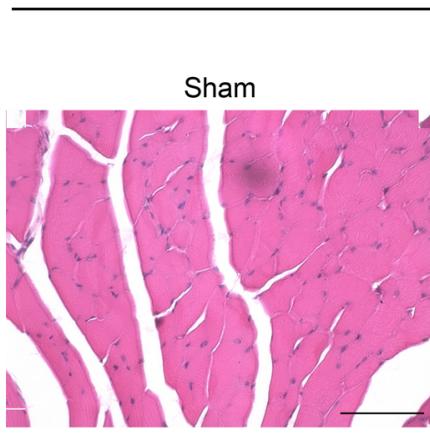

(n)
Foxp3-1 Syt7 $7^{-1-}$ adoptive transfer

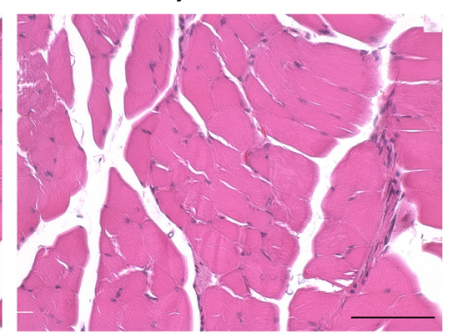

4 wk

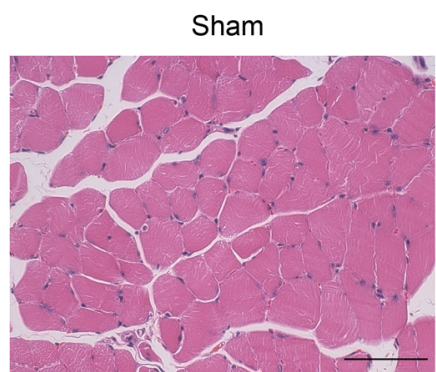

Foxp3-1 Syt7 ${ }^{-1-}$

adoptive transfer

Figure 1. Distal skeletal muscle is spared from inflammation in an adoptive transfer model of IIM. Representative images of H\&E-stained skeletal muscles from Rag $1^{-/-}$mice receiving sham adoptive transfer or adoptive transfer of lymph node cells from Foxp $3^{-/ \gamma}$ Syt7 $7^{-1-}$ mice. Rag $1^{-1-}$ mice receiving adoptive transfer of lymph node cells from the Foxp $3^{-/ \gamma}$ Syt7 $7^{-/-}$background have large areas of inflammation and fatty tissue infiltrates in proximal muscle. (A) Quadriceps muscle from sham and adoptive transfer mice at 1 and 4 weeks after adoptive transfer. (B) EDL muscle from sham and adoptive transfer mice at 1 and 4 weeks after adoptive transfer. Scale bars: $200 \mu \mathrm{m}$. Images are representative of 4 mice per group.

Foxp $3^{-/ Y}$ mice lack the critical transcription factor forkhead box P3 that acts as a master regulator responsible for the maturation and activation of Tregs (45) and develop severe lymphoproliferative and myeloproliferative disease within a few weeks after birth (46, 47). Syt $7^{-/-}$mice have a targeted disruption in the C2A calciumbinding domain of the Syt7 gene, resulting in decreased lysosomal exocytosis and plasma membrane resealing (10, 13, 48-50). Intact flexor digitorum brevis (FDB) muscles were dissected and intact individual muscle fibers were injured by irradiation with an infrared laser in the presence of the lipophilic dye FM4-64 to quantify the extent of sarcolemmal resealing in each genetic background. As previously demonstrated by others $(10,17)$, Syt $7^{-/-}$mice had a severe sarcolemmal resealing defect. An approximately 4.6fold increase in FM4-64 dye uptake was observed compared with C57BL controls (Figure 3, A-C), indicative of defective sarcolemmal resealing. Foxp $3^{-/ Y}$ mice did not show a significant difference in total dye uptake versus C57BL control FDB muscles (Figure 3, $\mathrm{A}-\mathrm{C}$ ), indicating that the lack of Tregs in the Foxp3 $3^{-/ Y}$ background is not sufficient to induce a sarcolemmal resealing defect.

Adoptive transfer of lymph node cells from Foxp $3^{-/ Y}$ Syt $7^{-/-}$doublemutant mice significantly impairs sarcolemmal resealing capacity. The observation of decreased sarcolemmal integrity suggests that membrane resealing may be compromised in IIM. Decreased sarcolemmal integrity in the absence of inflammation seen in distal skeletal muscle represents an aberrant source of intramuscular antigens. We did not observe a significant decrease in sarcolemmal resealing in Foxp $3^{-/ Y}$ FDB muscles and expanded our studies to determine whether priming of lymphocytes in the doublemutant background lacking mature Tregs combined with the exposure of intramuscular antigens due to the $S y t 7$ C2A calciumbinding domain mutation would interfere with normal sarcolemmal repair in recipient mice following adoptive transfer. Western blots of serum creatine kinase (CK) levels in $\mathrm{Ragl}^{-/-}$mice receiving adoptive transfer of lymph node cells from Foxp $3^{-/ Y} \mathrm{Syt}^{-/-}$mice collected at 1 and 4 weeks after adoptive transfer indicated that circulating CK was elevated, although not to a statistically significant degree (Supplemental Figure 4). We also examined TRIM72 protein expression in sham and adoptive transfer mice and observed a significant increase in TRIM72 in the gastrocnemius muscles of adoptive transfer mice and no significant change in expression in soleus and EDL muscles (Supplemental Figure 5). TRIM72 is a critical component of sarcolemmal resealing in skeletal muscle (37, 38,41 , and the observed increase in protein expression in the gastrocnemius may represent a compensatory response to a decreased ability of the sarcolemma to reseal after insult.

To elucidate whether and to what extent membrane resealing is compromised, we performed a laser-induced injury assay on intact FDB muscles isolated from sham Rag1 $^{-/-}$mice and $\mathrm{Rag1}^{-{ }^{--}}$mice 
A
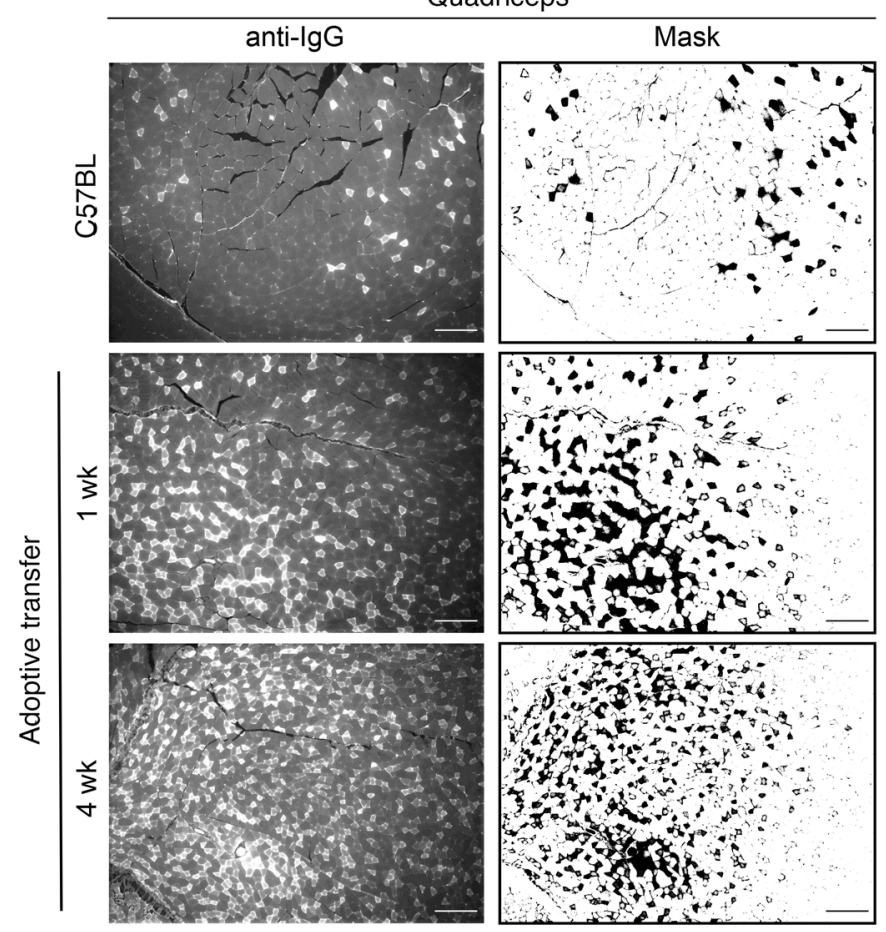

C

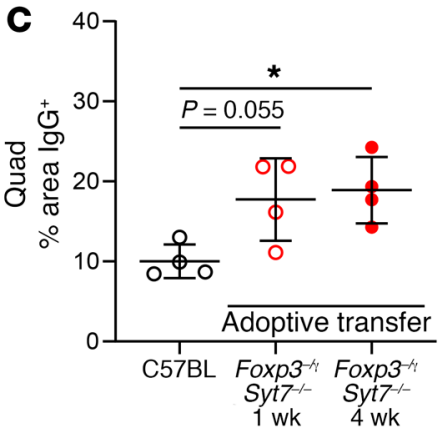

B
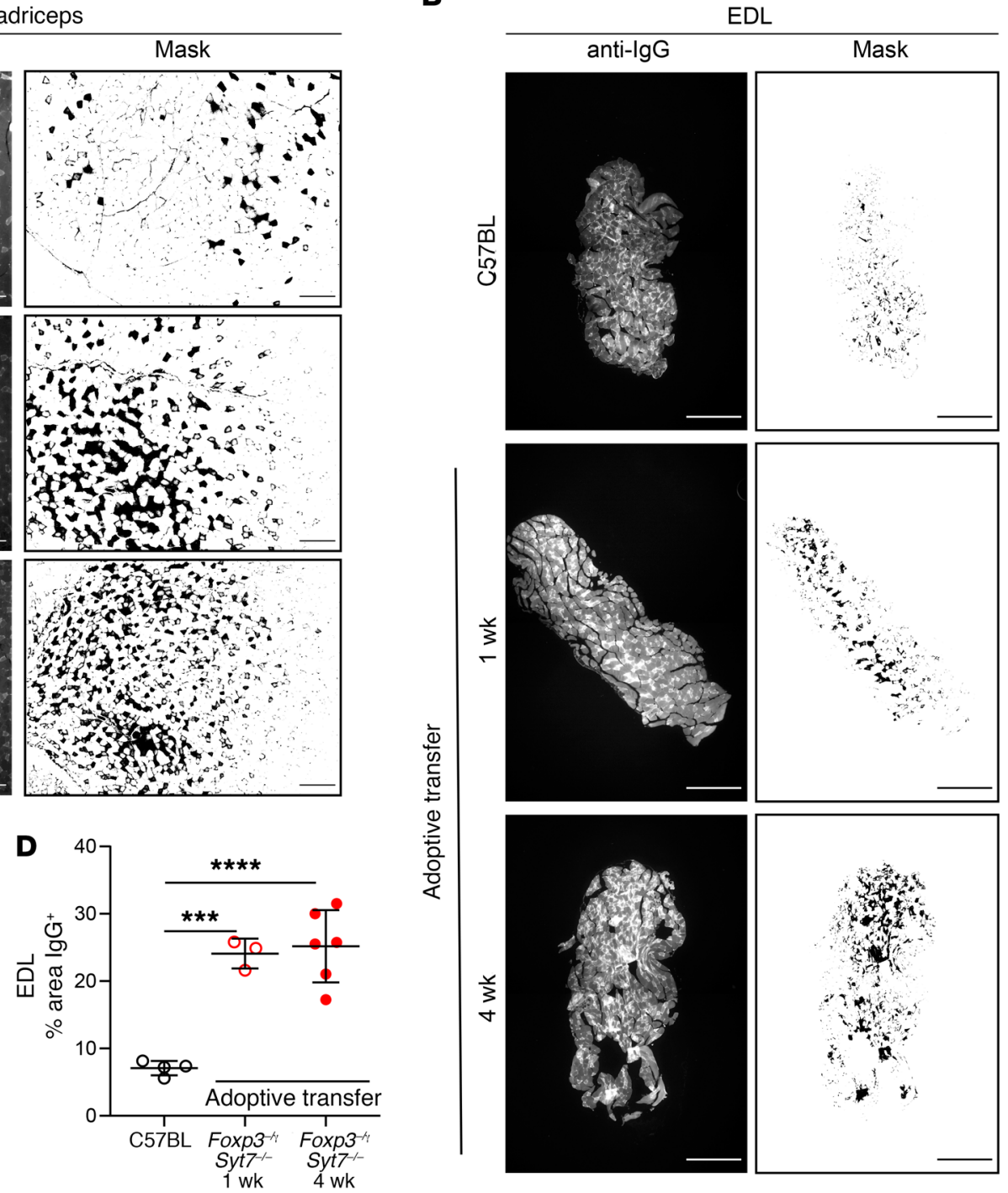

Figure 2. Membrane integrity is significantly reduced in proximal and distal muscle in an adoptive transfer model of IIM. (A) Representative images of IgG-labeled quadriceps from C57BL and Rag1 $1^{-/-}$mice receiving adoptive transfer from Foxp3 $3^{-/ \gamma}$ Syt7 $7^{-/-}$mice. Scale bars: $200 \mu \mathrm{m}$. (B) Representative images of IgG-labeled EDL from C57BL and from Rag1 $1^{-/-}$mice receiving adoptive transfer from Foxp $3^{-/ Y}$ Syt7 $7^{-/-}$mice. Scale bars: $200 \mu \mathrm{m}$. (C) Quantification of quadriceps percentage area positive for IgG ( $n=4$; ANOVA, $F_{(2,9)}=5.801, P=0.0241$; Tukey's honestly significant difference [HSD]: C57BL vs. 1 week adoptive transfer, $P=0.0556$; C57BL vs. 4 weeks adoptive transfer, $P=0.0294$ ). Data are represented as mean \pm SD. (D) Quantification of EDL percentage area positive for IgC $\left(n=4,3\right.$, and 6 for $C 57 \mathrm{BL}$, adoptive transfer 1 week, and adoptive transfer 4 weeks, respectively; ANOVA, $F_{(2,10)}=27.77, P<0.0001 ;$ Tukey's HSD: C57BL vs. 1 week adoptive transfer, $P=0.0006$; C57BL vs. 4 weeks adoptive transfer, $P<0.0001)$. Data are represented as mean \pm SD.

receiving adoptive transfer of lymph node preparations from either Foxp $3^{-/ Y}$ mice or Foxp $3^{-/ Y}$ Syt7-/ double-mutant mice 1 week and 4 weeks after adoptive transfer. FDB muscle fibers from Rag1 ${ }^{-}$mice receiving adoptive transfer of lymph node cells from the Foxp $3^{-/ Y}$ Syt7-- background showed a 4.8-fold increase in total FM4-64 dye influx in comparison with sham adoptive transfer Rag1/- mice after 1 week (Figure 4, A-C, and Supplemental Figure 6).

At 4 weeks after adoptive transfer, FM4-64 dye uptake in Foxp $3^{-/ Y}$ adoptive transfer mice remained unchanged from that in sham controls (Figure 4, A-C). Conversely, Rag1 ${ }^{-/}$mice adoptively transferred with lymph node cells from the Foxp $3^{-/ Y} \mathrm{Syt}^{-1-}$ double-mutant background showed a 4.75 -fold increase in total FM4-64 dye uptake at 4 weeks (Figure 4, B and C). These data demonstrate that priming of lymphocytes in the double-mutant genetic background decreases sarcolemmal resealing capacity in the absence of the Syt7 mutation. Exposure of intramuscular antigens to a Treg-deficient milieu due to an underlying defect in sarcolemmal resealing may be a contributing factor in pathogenesis and may exacerbate the progression of IIM.

Autoantibodies against TRIM72 are elevated in IIM subject sera and Foxp $3^{-1 Y}$ Syt7 $7^{-1}$ double-mutant mouse sera. We observed decreased sarcolemmal integrity and decreased sarcolemmal resealing in the absence of inflammation in our adoptive transfer model of IIM. Taken together, our findings imply that a mechanism other than direct immune cell-mediated skeletal muscle damage is affecting sarcolemmal integrity and resealing in distal skeletal muscle. We hypothesized that proteins involved in sarcolemmal resealing will routinely become exposed in an aberrant manner 
A
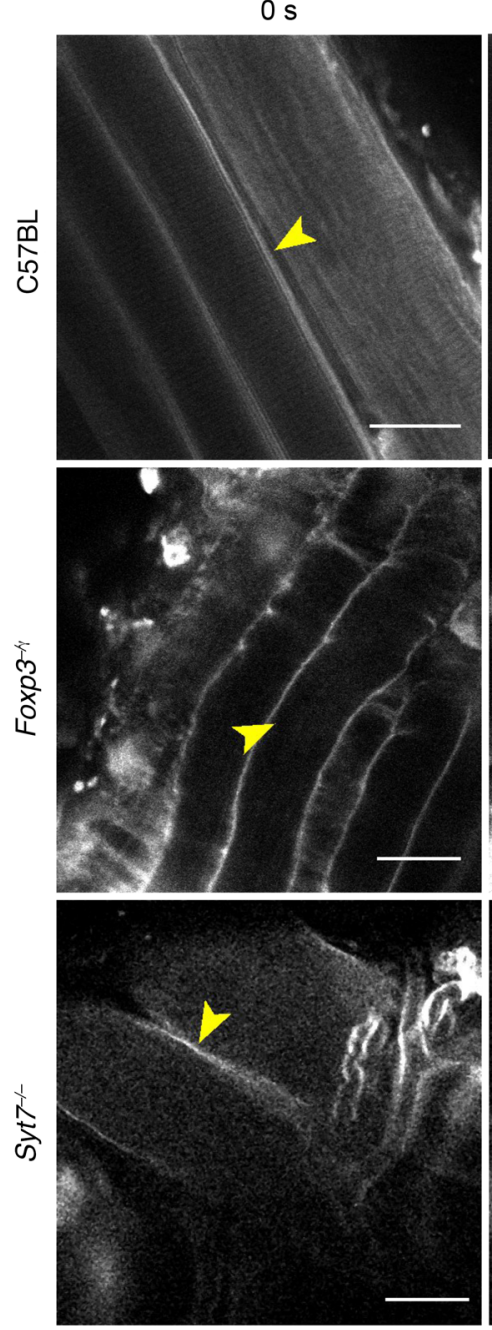

$60 \mathrm{~s}$
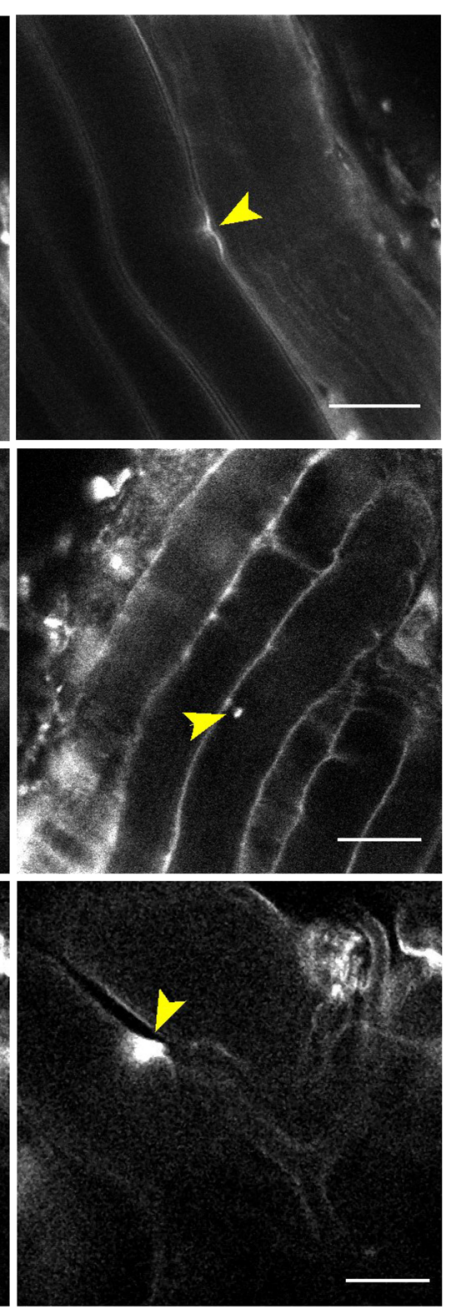

B

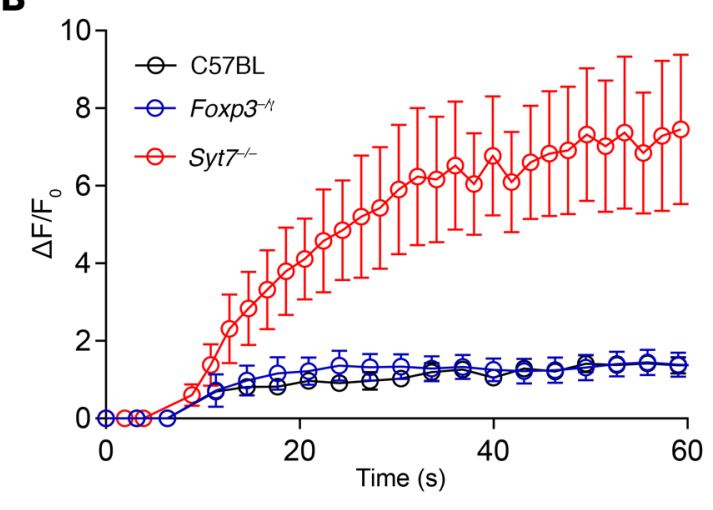

C

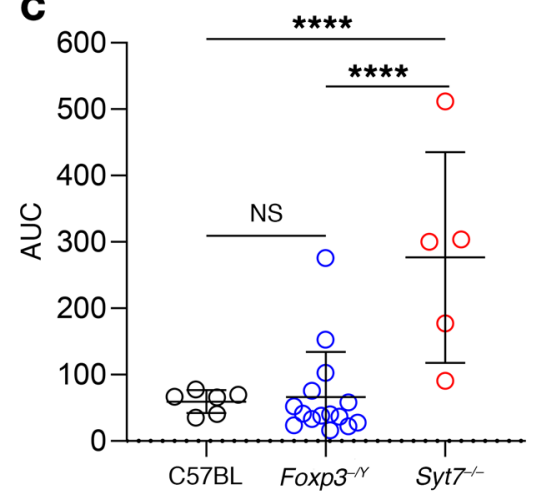

Figure 3. Treg deficiency is not sufficient to compromise sarcolemmal resealing. FDB muscles isolated from indicated mice and injured with an infrared laser in the presence of FM4-64 dye. The kinetics of sarcolemma resealing was measured by acquisition of images every 3 seconds for 60 seconds and calculation of the change in fluorescence before and after injury. (A) Representative images of C57BL, Foxp $3^{-1 /}$, and Syt7 $7^{-1-}$ FDB muscles before ( 0 seconds) and after (60 seconds) injury. Scale bars: $20 \mu \mathrm{m}$. Yellow arrowheads indicate sites of injury. (B) Curves depicting mean sarcolemmal resealing kinetics measured every 3 seconds for 60 seconds. Data are represented as mean \pm SEM. (C) AUC calculations representing total dye influx over time $(n=6,15$, and

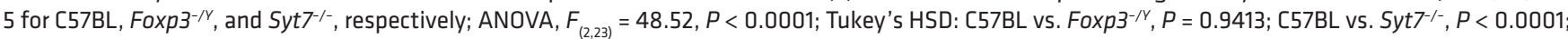
Syt7 $7^{-1-}$ vs. Foxp $\left.3^{-/ y}, P<0.0001\right)$. Data are represented as mean $\pm S D$.

and could be a source of immunogenicity, thus triggering an autoimmune response and the generation of autoantibodies. TRIM72 is an established protein involved in sarcolemmal resealing (37-39), and because we observed increased TRIM72 protein expression in the gastrocnemius muscles of our adoptive transfer model of IIM (Supplemental Figure 5), we examined TRIM72 expression in human skeletal muscle biopsies from healthy control, statininduced necrotizing myopathy (SINM), and dermatomyositis (DM) subjects by Western blot. TRIM72 was expressed at significantly higher levels in DM skeletal muscle versus healthy controls (Supplemental Figure 7). The observed increase in TRIM72 protein levels can be interpreted as a compensatory response to sarcolemmal damage that may promote its potential as an autoantigen in IIM.

We screened for TRIM family proteins that may act as autoantigens in IIM using a Western blot-based assay. Briefly, HEK293 cells were transfected to express TRIM family clones of interest tagged with eGFP. Cell lysates were enriched by immuno-pulldown using the eGFP tag. Enriched lysates were separated by SDS-PAGE, and Western blots were performed to detect TRIM family autoantigens using IIM subject serum as the primary antibody. We tentatively identified 6 TRIM family proteins as potential autoantibody targets in IIM subject sera (Supplemental Figure 8), with autoantibodies against TRIM72 appearing in several subject serum samples. We specifically chose to focus on TRIM72 because of its established critical function in sarcolemmal resealing and to test the mechanistic role TRIM72 autoantibodies may have in the progression of IIM. We validated the presence of TRIM72 autoantibodies in our adoptive transfer model of myositis by Western blot and dot blot. We observed signals corresponding to autoantibodies targeting TRIM72 in Foxp $3^{-/ Y}$ Syt $^{-/}$adoptive transfer sera using 
A

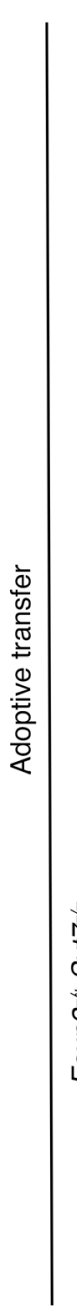

$0 \mathrm{~s}$
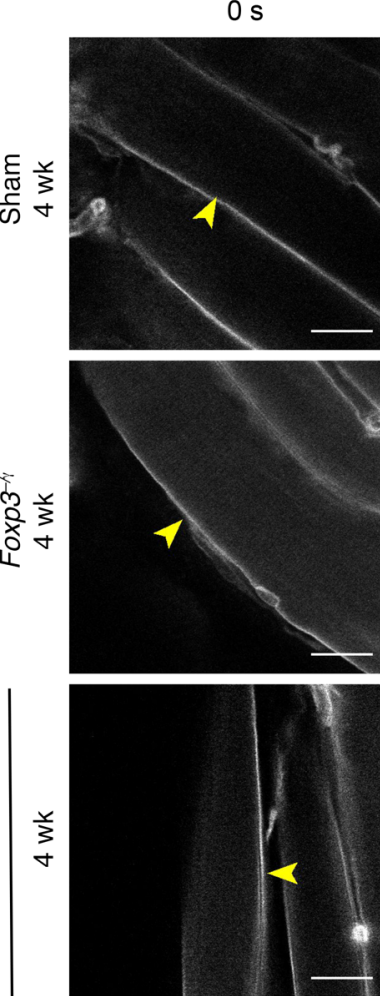

ڤ్

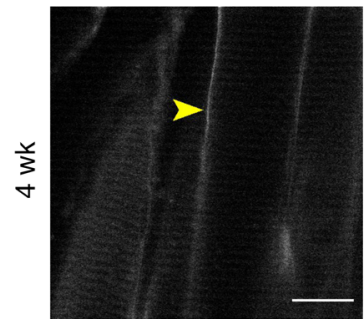

$60 \mathrm{~s}$
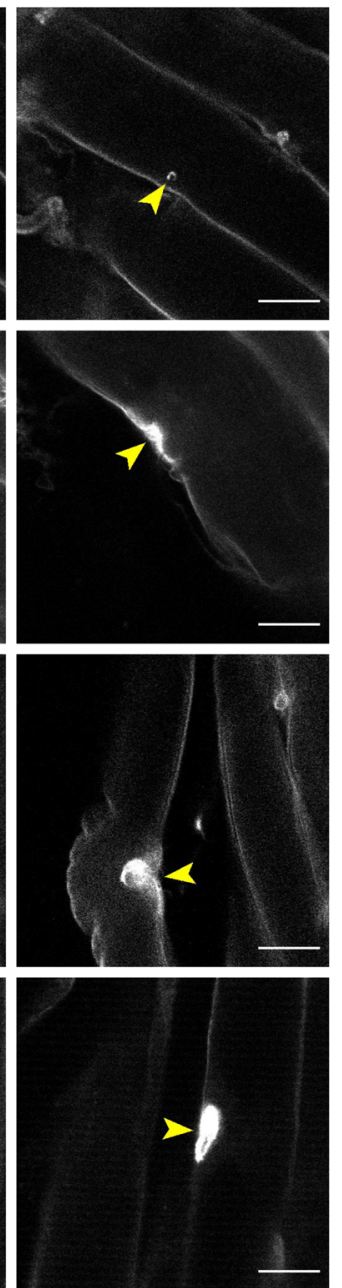

B

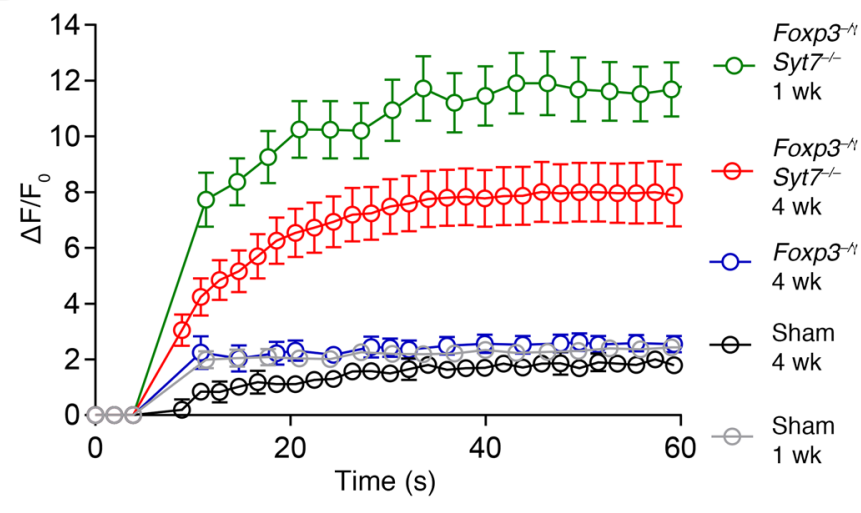

C
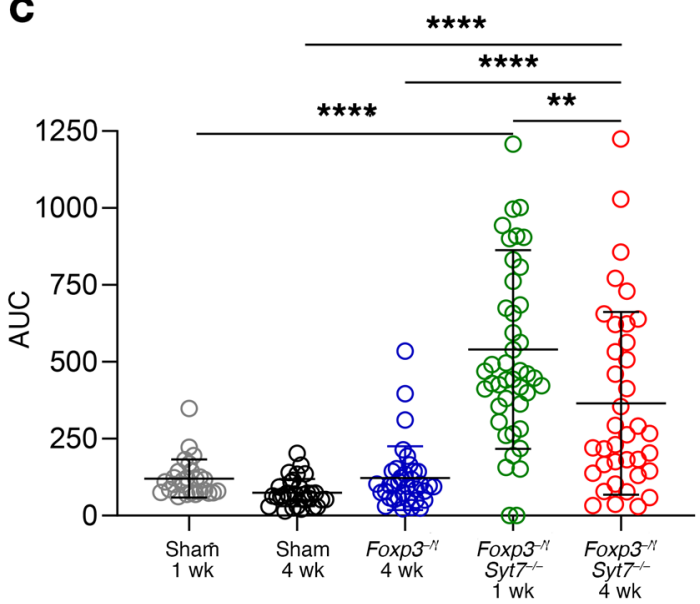

Figure 4. Sarcolemmal resealing capacity is significantly diminished following adoptive transfer of lymph node cells from Foxp $3^{-/ \gamma}$ Syt $7^{-/-}$mice. FDB muscles isolated from indicated mice and injured with an infrared laser in the presence of FM4-64 dye. The kinetics of sarcolemma resealing was measured by acquisition of images every 3 seconds for 60 seconds and calculation of the change in fluorescence before and after injury. (A) Representative

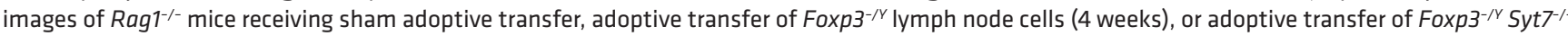
lymph node cells (1 week and 4 weeks). Scale bars: $20 \mu \mathrm{m}$. Yellow arrowheads indicate sites of injury. (B) Curves depicting mean sarcolemmal resealing kinetics measured every 3 seconds for 60 seconds. Data are represented as mean \pm SEM. (C) AUC calculations representing total dye influx over time (Sham 1 week, $n=27$; Sham 4 weeks, $n=31$; Foxp3 ${ }^{-/ \gamma}$ adoptive transfer, $n=37$; Foxp3 $3^{-/ \gamma}$ Syt7 $7^{-/-}$adoptive transfer 1 week and 4 weeks, $n=44$ and 37, respectively; ANOVA, $F_{(4.171)}=28.47, P<0.0001$; Tukey's HSD: Sham 1 week vs. Foxp $3^{-/ \gamma}$ Syt7 $7^{-1-} 1$ week, $P<0.0001$; Sham 4 weeks vs. Foxp $3^{-/ \gamma}$ Syt7 $7^{-1-} 4$ weeks, $P<$ $0.0001 ;$ Foxp3 $^{-/ /} 4$ weeks vs. Foxp3 $3^{-/ Y}$ Syt7 $7^{-/-} 4$ weeks, $\left.P=0.0005\right)$. Data are represented as mean \pm SD.

both methods and observed little or no expression in Foxp $3^{-1 Y}$ or C57BL serum samples (Supplemental Figure 9).

To increase the fidelity of our analysis, we developed (Supplemental Figure 10) an ELISA to detect autoantibodies targeting TRIM72. Autoantibodies against TRIM72 were significantly increased in Foxp $3^{-1 Y}$ Syt7 $7^{-/}$mice compared with C57BL. While autoantibodies in the Foxp $3^{-/ Y}$ background were elevated, levels were not significantly different from observed levels in C57BL mice (Figure 5A).

Rag1 $1^{-/-}$mice receiving adoptive transfer of Foxp $^{-/ Y}$ lymph node cells did not have TRIM72 autoantibody levels above those detected in sham adoptive transfer mice (Figure 5B). One week after adoptive transfer, Rag1 ${ }^{-/}$mice receiving adoptive transfer of lymph node cells from the Foxp $3^{-/ Y}$ Syt $7^{-/-}$double-mutant back- ground had significantly elevated levels of TRIM72 autoantibodies. Although TRIM72 autoantibody levels were not significantly elevated 4 weeks after adoptive transfer owing to within-group variance, autoantibodies were considerably elevated in 4 of the 9 mouse sera tested (Figure 5B). The observed within-group variance may be partially explained by the serum half-life of mouse IgG (6-8 days; refs. 51-53) and the low number of B cells that persist at 4 weeks after adoptive transfer (12).

We also used our custom ELISA to screen 103 IIM subject serum samples (DM, $n=52$; PM, $n=51$; healthy, $n=15$ ) for autoantibodies against TRIM72 and identified a substantial subset of sera with elevated levels of TRIM72 autoantibodies. Using a cutoff value of $1 \mathrm{SD}$ above the mean determined for healthy subject sera (mean $+1 \mathrm{SD}=0.56 \mathrm{OD}$ reading), $34.6 \%$ and $21.6 \%$ of DM and 


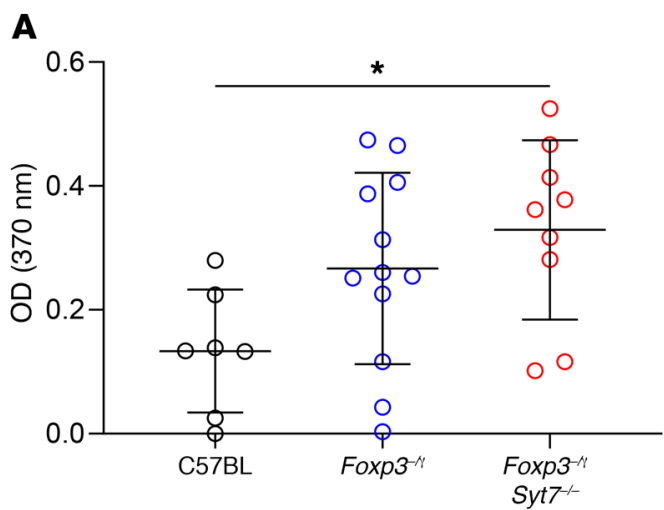

C

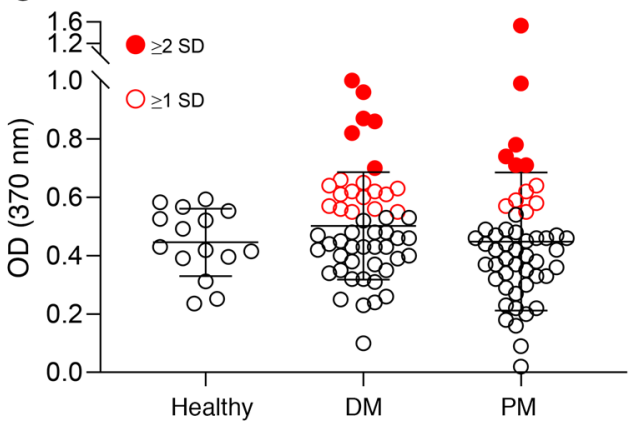

B

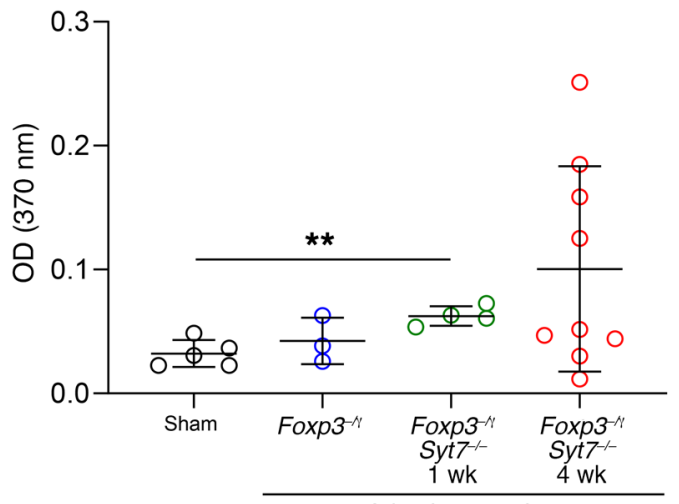

Adoptive transfer

Figure 5. TRIM72 autoantibodies are elevated in Foxp3 $3^{-1 /}$ Syt7--- double-mutant mice, mice receiving adoptive transfer of lymph node cells from

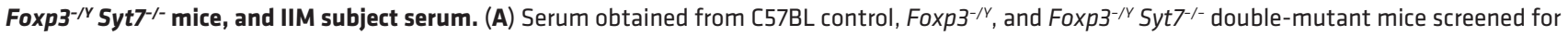
TRIM72 autoantibodies by ELISA $\left(n=8,13\right.$, and 9 for C57BL, Foxp3 $3^{-/ Y}$, and Foxp3 $3^{-/ Y}$ Syt7 $7^{-/-}$mice, respectively; ANOVA, $F_{(2,25)}=3.945, P=0.0324$; Dunnett's test: C57BL vs. Foxp $3^{-/ Y}$ Syt7 $7^{-1}, P=0.0187$ ). (B) Serum from sham adoptive transfer, Foxp $3^{-/ \gamma}$ lymph node cell adoptive transfer, and Foxp $3^{-/ Y}$ Syt7 $7^{-/-}$ double-mutant lymph node cell adoptive transfer screened for TRIM72 autoantibodies by ELISA ( $n=5,3,4$, and 10 for sham, Foxp3 $3^{-/ v}$, Foxp $3^{-/ v}$ Syt7 $7^{-1-1}$

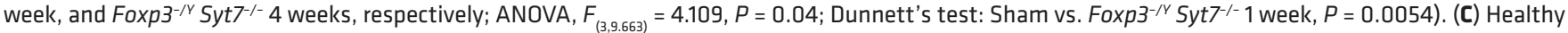
$(n=15)$, dermatomyositis (DM; $n=52)$, and polymyositis $(P M ; n=51)$ subject sera screened for TRIM72 autoantibodies by ELISA. Red dots indicate samples with OD values greater than 1 SD compared with healthy subject sera; $34.6 \%$ and $21.6 \%$, respectively, of DM and PM subject serum screened had OD values greater than 1 SD compared with healthy subject serum. Data in $\mathbf{A}-\mathbf{C}$ are represented as mean \pm SD.

PM subject sera tested were positive for elevated levels of TRIM72 autoantibodies (Figure 5C). Using a more conservative cutoff value of $2 \mathrm{SD}$ (mean $+2 \mathrm{SD}=0.67 \mathrm{OD}$ reading), $11.5 \%$ and $11.8 \%$ of DM and PM subject sera had elevated TRIM72 autoantibody levels. Available clinical subject data are summarized in Table 1.

Exogenous delivery of polyclonal TRIM72 antibody significantly impairs sarcolemmal resealing. Intact FDB skeletal muscles from C57BL mice were exposed to exogenous anti-TRIM72 antibodies (dilutions of 1:300 and 1:100 from a $2-\mathrm{mg} / \mathrm{mL}$ anti-TRIM72 stock) and then subjected to laser-induced injury to determine whether the binding of antibodies hinders repair patch formation. Antibodies against TRIM72 significantly reduced sarcolemmal resealing in a concentration-dependent manner (Figure 6, A-D). Area under the curve (AUC) calculations to determine the total dye influx over time revealed a 4.0- and 5.7-fold increase of FM464 dye influx for 1:300 and 1:100 dilutions of TRIM72 antibody, respectively (Figure 6D). We confirmed our findings using 2 additional membrane damage assays. HEK293 cells were transfected with either eGFP or TRIM72 constructs and injured with silica beads using a rotational damage assay. Lactate dehydrogenase $(\mathrm{LDH})$ release from injured cells was quantified as a measure of membrane resealing capacity. HEK293 cells expressing TRIM72 released significantly less LDH than eGFP-transfected cells as expected (Figure 6E; eGFP +Injury -Ab vs. TRIM72 +Injury -Ab). The addition of polyclonal TRIM72 antibody to eGFP control cells had no significant effect on LDH release (Figure 6E; eGFP +Injury -Ab vs. eGFP +Injury +Ab). Importantly, HEK293 cells expressing TRIM72 injured in the presence of antibody released significantly more $\mathrm{LDH}$ versus rabbit serum control (Figure 6E; TRIM72 +Injury -Ab vs. TRIM72 +Injury +Ab). As a tertiary means of confirmation, $\mathrm{C} 2 \mathrm{C} 12$ myoblasts were differentiated into myotubes by serum starvation to induce expression of TRIM72. After 5-7 days of serum starvation, differentiated myotubes were injured with $600-\mu \mathrm{m}$ silica beads on an orbital shaker in the presence of normal rabbit serum or a 1:300 dilution (from $2-\mathrm{mg} / \mathrm{mL}$ stock) of anti-TRIM72 antibody. Myotubes injured with antibodies against TRIM72 released approximately 3-fold higher levels of LDH compared with rabbit serum controls (Supplemental Figure 11). These findings demonstrate that antibody neutralization of TRIM72 can negatively affect sarcolemmal resealing after injury.

IIM subject sera with elevated levels of TRIM72 autoantibodies suppress sarcolemmal resealing in healthy skeletal muscle. To determine whether IIM subject sera with elevated levels of TRIM72 autoantibodies can impair sarcolemmal resealing in healthy skeletal muscle, we subjected FDB muscles from C57BL mice to laser injury in the presence of IIM subject serum determined to have 
Table 1. Subject serum myositis autoantibodies and CPK levels

\begin{tabular}{|c|c|c|c|}
\hline Autoantibody & DM subject sera & PM subject sera & Elevated serum CPK \\
\hline TRIM72A $^{\mathrm{A}}$ & $34.6(18)$ & $21.6(12)$ & $57.9(11)$ \\
\hline Jo-1 & $10.7(3)$ & $40.6(13)$ & $61.5(8)$ \\
\hline $\mathrm{PL}-12^{\mathrm{B}}$ & $7.1(2)[7.1(2)]^{\mathrm{B}}$ & $3.1(1)$ & - \\
\hline TIF1 $1 \gamma^{\mathrm{B}}$ & $28.6(8)[10.7(3)]^{\mathrm{B}}$ & $3.1(1)$ & - \\
\hline $\mathrm{Mi}-2^{\mathrm{B}}$ & $14.3(4)[3.6(1)]^{\mathrm{B}}$ & $3.1(1)$ & $100(2)$ \\
\hline SAE & $3.6(1)$ & - & - \\
\hline Ro60 & $3.6(1)$ & - & $100(1)$ \\
\hline MJ & $7.1(2)$ & $3.1(1)$ & $100(1)$ \\
\hline U1RNA ${ }^{B}$ & $3.6(1)$ & $3.1(1)[3.1(1)]^{\mathrm{B}}$ & 50 (1) \\
\hline Ro52 ${ }^{\mathrm{B}}$ & - & $3.1(1)[3.1(1)]^{\mathrm{B}}$ & - \\
\hline $\mathrm{SRP}^{\mathrm{B}}$ & - & $6.3(2)[3.1(1)]^{\mathrm{B}}$ & - \\
\hline SSA & - & $3.1(1)$ & - \\
\hline Unidentified protein ${ }^{B}$ & - & $3.1(1)[3.1(1)]^{\mathrm{B}}$ & - \\
\hline Negative $^{B}$ & $25.0(7)[3.6(1)]^{\mathrm{B}}$ & $28.1(9)[6.3(2)]^{B}$ & $57.1(8)[28.6(4)]^{\mathrm{B}}$ \\
\hline
\end{tabular}

Values represent the percentage of samples positive for a given myositis autoantibody with positive $n$ values in parentheses. All values are representative of available clinical subject data for each category. ${ }^{A}$ Samples indicated for TRIM72 autoantibodies were determined by ELISA. ${ }^{B}$ Autoantibodies and corresponding percentages with $n$ values listed in brackets represent serum samples positive for the indicated myositis autoantibody and elevated levels of TRIM72 autoantibodies determined by ELISA as described in Methods. CPK, creatine phosphokinase.
Depletion/reduction of TRIM72 autoantibodies from IIM subject sera rescues compromised sarcolemmal resealing in healthy skeletal muscle. To establish a direct effect of TRIM72 autoantibodies on the compromised sarcolemmal resealing observed in healthy skeletal muscle injured in the presence of IIM subject sera, we depleted/reduced TRIM72 autoantibodies. Briefly, NHS-activated magnetic beads were conjugated with full-length recombinant human TRIM72 (rhTRIM72) protein and incubated with subject sera determined to have elevated levels of TRIM72 autoantibodies by ELISA. Beads were then collected with a magnetic stand, and the TRIM72 autoantibody-depleted serum was collected. Captured TRIM72 autoantibodies were eluted from magnetic beads with $0.1 \mathrm{M}$ glycine $(\mathrm{pH}$ 2.0). Isolation and purification of TRIM72 autoantibodies from IIM subject sera yielded $127.3 \mu \mathrm{g} / \mathrm{mL}, 147.2 \mu \mathrm{g} / \mathrm{mL}$, and $251.3 \mu \mathrm{g} / \mathrm{mL}$ from IIM serum samples 1-3, respectively (Supplemental Figure 15). The sarcolemma of isolated FDB muscles from C57BL mice was injured using an infrared laser as previelevated levels of TRIM72 autoantibodies. Each muscle bundle was used as its internal control ("Basal") to establish normal sarcolemmal resealing kinetics followed by a second round of laser injury on previously uninjured muscle fibers with exogenously added IIM subject serum ("Serum") at a dilution of 1:200. Serum from healthy control subjects did not affect sarcolemmal resealing kinetics (Figure 7, A and B), and AUC measurements revealed no change in total dye uptake over time (Figure 7C). The addition of IIM subject sera with elevated levels of TRIM72 autoantibodies significantly reduced sarcolemmal resealing capacity regardless of the IIM subtype tested. The addition of PM subject serum significantly increased FM4-64 dye influx in healthy skeletal muscle (Figure 7, D and E), as indicated by a 3.6-fold increase in AUC measurements of total dye influx over time (Figure 7F). DM subject serum had a similar detrimental effect on sarcolemmal resealing (Figure 7, G-I), with a 2.3-fold increase in total dye influx.

As a secondary means of validation, we performed 2 additional injury assays to determine the effect of IIM subject serum on sarcolemmal resealing. Using a ballistic injury assay, gold microparticles were used to injure differentiated $\mathrm{C} 2 \mathrm{C} 12$ myotubes known to express TRIM72. We observed a trend of increased LDH release in myotubes injured with the addition of IIM subject sera versus healthy subject serum (Supplemental Figure 12B). We also found that both low and high levels of TRIM72 autoantibodies (defined as $>1 \mathrm{SD}$ and $>2 \mathrm{SD}$, respectively, by ELISA) significantly reduced membrane resealing capacity in our rotational damage assay using HEK293 cells expressing TRIM72 (Supplemental Figure 12A). Taken together, these data support our hypothesis that autoantibodies targeting TRIM72 decrease sarcolemmal resealing and potentially drive IIM progression by increasing aberrant intramuscular antigen exposure to an autoreactive immune system. ously described before experimental manipulation ("Basal") and with exogenous delivery of 1:300 dilutions of IIM subject sera ("IIM sera"), sera depleted of TRIM72 autoantibodies ("Depleted serum"), and isolated and purified TRIM72 autoantibodies from IIM subject sera ("Purified Hu anti-TRIM72"). Laser injury assays using human anti-TRIM72 purified from IIM subject sera were conducted using the same concentration of polyclonal antiTRIM72 represented by the 1:300 dilution in Figure $6(6.6 \mu \mathrm{g} / \mathrm{mL})$.

Sarcolemmal resealing was significantly impaired in the presence of exogenous IIM subject sera with elevated levels of TRIM72 autoantibodies (Basal vs. IIM serum, $P<0.0001$ ). Similarly, laser injury in the presence of purified TRIM72 autoantibodies from IIM subject sera resulted in significantly impaired sarcolemmal resealing capacity (Basal vs. Purified Hu anti-TRIM72, $P$ $<0.0001)$. Depletion/reduction of TRIM72 autoantibodies from IIM subject sera rescued the diminished resealing capacity in healthy skeletal muscle (Basal vs. Depleted serum, $P=0.95$; Figure $8, \mathrm{~A}-\mathrm{C}$; data for individual IIM serum samples are presented in Supplemental Figure 15). This finding directly implicates autoantibodies targeting sarcolemmal resealing and repair proteins, specifically TRIM72, as a driving factor in our observation of decreased sarcolemmal integrity and resealing in IIM and represents a pathogenic mechanism driving disease progression.

\section{Discussion}

Idiopathic inflammatory myopathies (IIM) are a group of rare disorders affecting skeletal muscle. This rarity and a lack of consensus regarding therapeutic targets and management of IIM have left a substantial gap in knowledge of the mechanisms leading to pathogenesis and progression of IIM. The majority of research, as well as the development of therapeutic strategies, has focused on immune cell-mediated cytotoxic damage and immune suppres- 
A
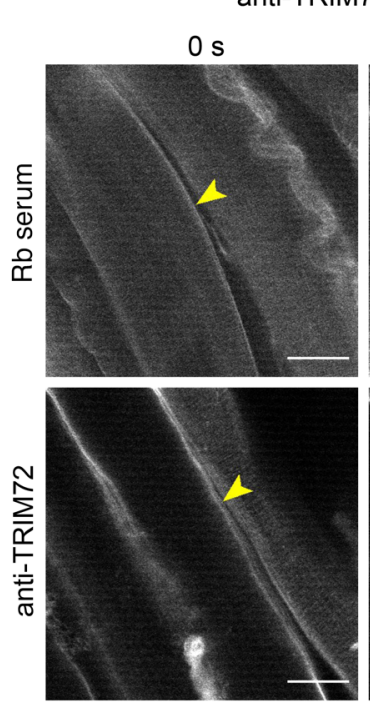

c

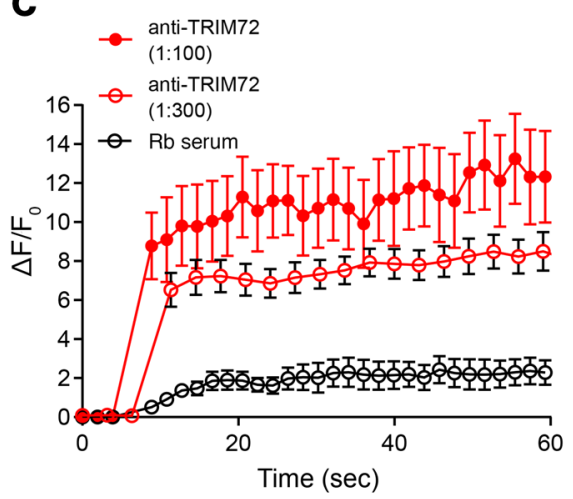

B

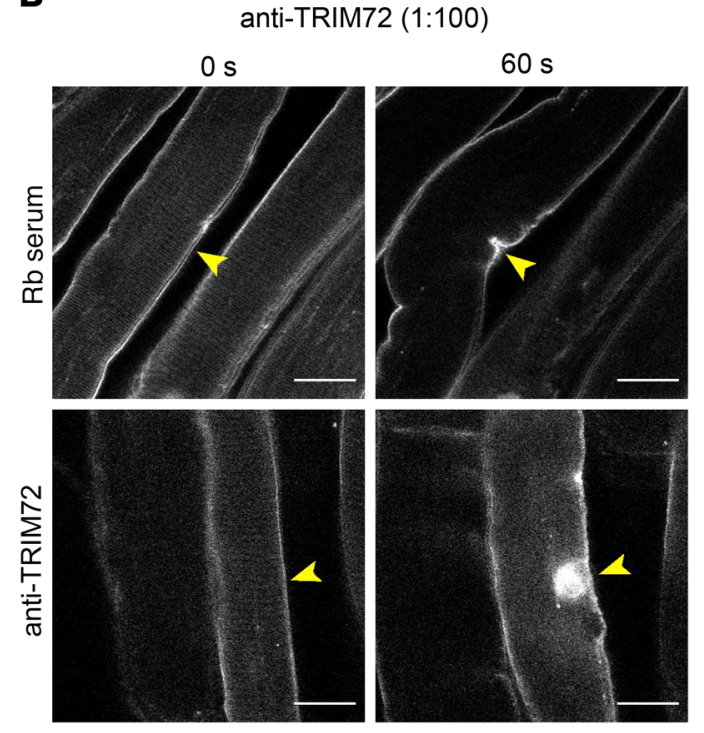

$60 \mathrm{~s}$
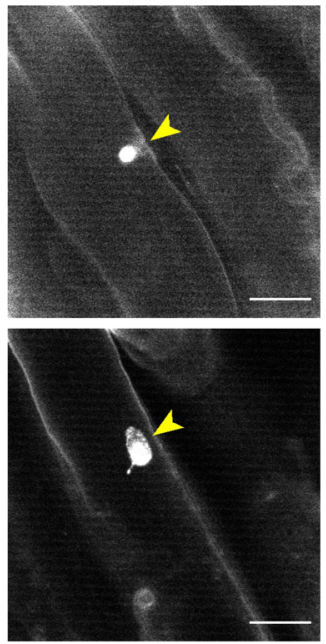

E

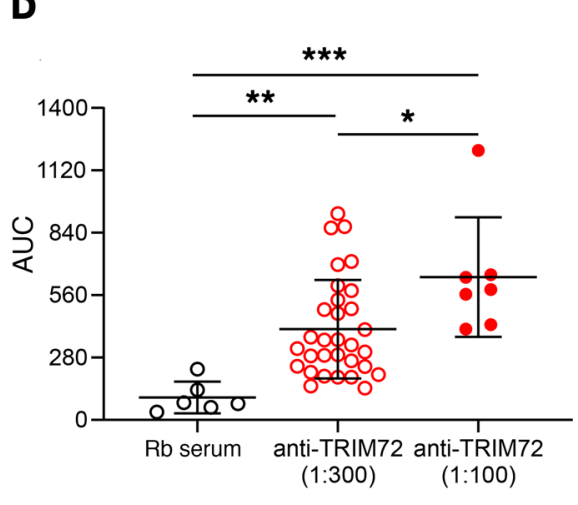

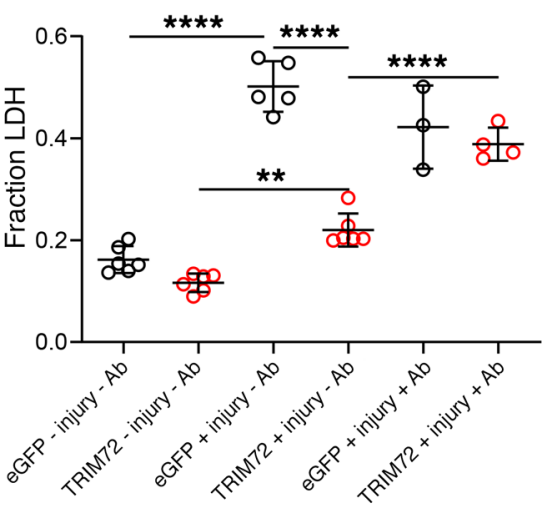

Figure 6. Exogenous delivery of polyclonal TRIM72 antibody significantly impairs sarcolemma resealing. FDB muscles isolated from C57BL mice were subjected to laser-induced injury with either normal rabbit $(\mathrm{Rb})$ serum (control, 1:100 dilution) or polyclonal antibody against TRIM72 (2 mg/mL diluted 1:300 and 1:100, respectively). Yellow arrowheads indicate sites of injury. (A) Representative images of FM4-64 dye influx before (0 seconds) and after (60 seconds) injury with anti-TRIM72 antibody at 1:300. (B) Representative images of FM4-64 dye influx before and after injury with anti-TRIM72 antibody at 1:100. Scale bars: $20 \mu \mathrm{m}$. (C) Curves depicting mean sarcolemmal resealing kinetics measured every 3 seconds for 60 seconds. Data are represented as mean \pm SEM. (D) AUC calculations representing total dye influx over time $(n=7,31$, and 7 for Rb serum, anti-TRIM72 at 1:300, and anti-TRIM72 at 1:100, respectively; ANOVA, $F_{(2.41)}=10.05, P=0.0003$; Tukey's HSD: Rb serum vs. 1:300, $P=0.0079$; Rb serum vs. 1:100, $P=0.0002 ; 1: 100$ vs. 1:300, $P=0.036$ ). (E) Rotation damage assay performed on HEK293 cells expressing eGFP (control) or TRIM72 with and without polyclonal antibody against TRIM72 (1:100 dilution). LDH release into the supernatant was quantified as a measure of membrane resealing capacity. (eGFP -Injury -Ab, $n=6$; eGFP +Injury -Ab, $n=5$; eGFP + Injury +Ab, $n=3$; TRIM72 -Injury -Ab, $n=6$; TRIM72 +Injury -Ab, $n=6$; TRIM72 +Injury + Ab, $n=4$. ANOVA, $F_{(5,24)}=80.74, P<0.0001 ;$ Tukey's HSD: ${ }^{* *} P<0.005,{ }^{* * *} P<0.0001$.) Data in $\mathbf{D}$ and $\mathbf{E}$ are represented as mean $\pm \mathrm{SD}$.

sion. Both innate and adaptive immune processes are known to have a role in IIM $(1,2,6,7,31,54-56)$; however, the mechanism responsible for initiation and progression of IIM remains elusive. Recent advances in our understanding of immune-mediated processes leading to skeletal muscle damage and the immunological dysfunction associated with IIM highlight the complexity and heterogeneity of this disease.

Disruption of the sarcolemma can be caused by bacterial and viral infections, trauma, and the physiological force of skeletal muscle contraction during exertion. We propose that a sarcolemmal resealing defect is an early event in the development of IIM. Here, we identify a mechanism responsible for the resealing defects observed in histologically normal skeletal muscle. Autoantibodies targeting the membrane resealing/ repair protein TRIM72 hinder vesicle nucleation at injury sites on the sarcolemma, preventing normal repair patch formation. Autoantibodies targeting critical proteins involved in the recruitment and nucleation of repair patch vesicles at the site of injury may potentially lead to skeletal muscle fibers with compromised membrane barrier function, providing a continuous source of autoantigens to promote autoimmunity and further amplifying humoral responses.

Our previous studies using an adoptive transfer mouse model of IIM indicate that while a deficiency in Treg cell function is required, 
A

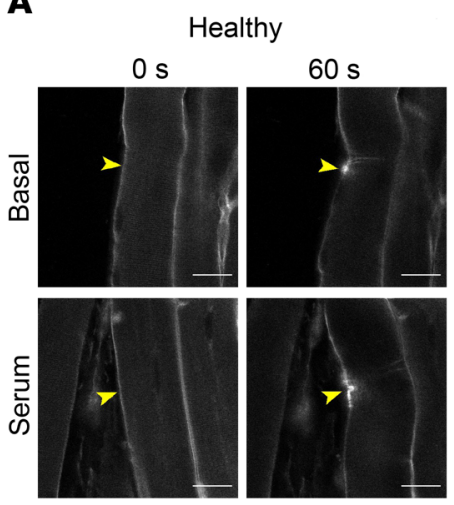

B

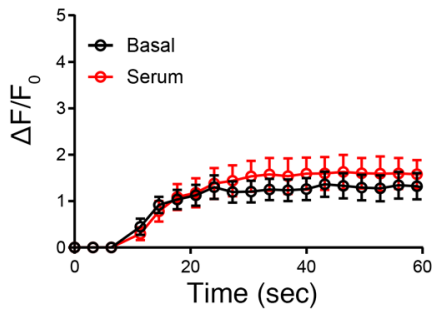

C

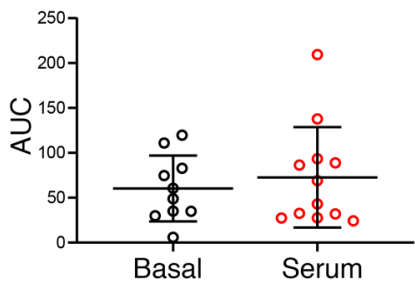

D

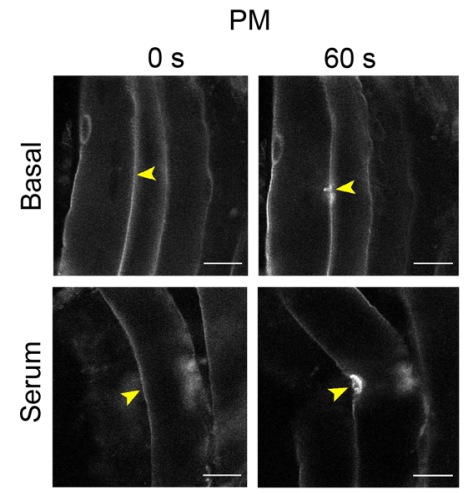

E

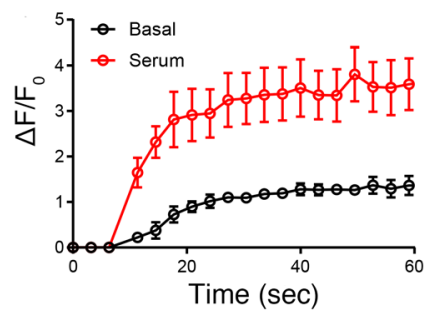

F

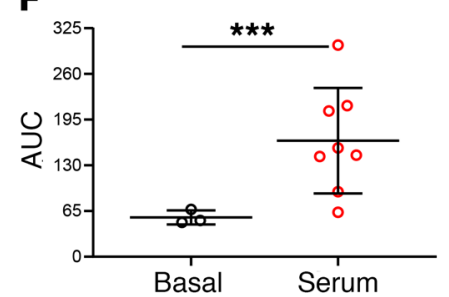

G

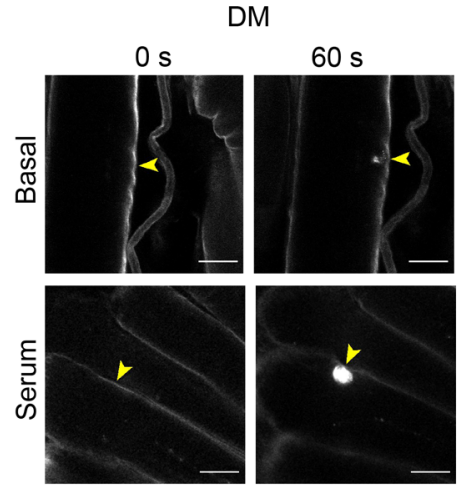

H

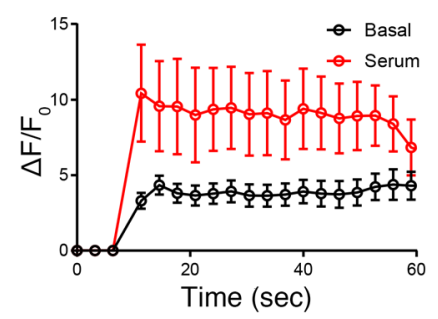

I

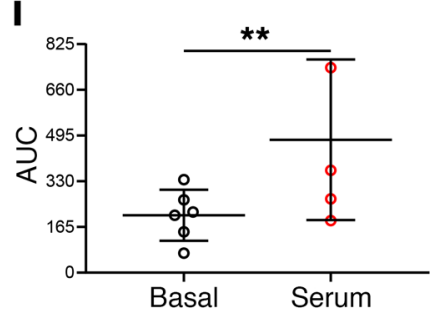

Figure 7. IIM sera with elevated TRIM72 autoantibody levels compromise sarcolemmal repair in healthy skeletal muscle. FDB muscles isolated from C57BL mice injured by an infrared laser with healthy subject serum or serum from subjects diagnosed with PM or DM. Serum was diluted 1:200 in Tyrode's buffer before laser injury. Yellow arrowheads indicate sites of injury. Data in $\mathbf{B}, \mathbf{E}$, and $\mathbf{H}$ are represented as mean \pm SEM. Data in C, F, and $\mathbf{I}$ are represented as mean \pm SD. (A) Representative images of FM4-64 dye influx before ( 0 seconds) and after ( 60 seconds) injury at baseline (Basal) or after addition of healthy subject serum (Serum). (B) Sarcolemmal resealing kinetics measured every 3 seconds for 60 seconds as depicted in A. (C) AUC calculations of B representing total dye influx over time $\left(n=10\right.$ and 12 for Basal and Serum, respectively; $t$ test, $\left.t_{(\mathrm{df}=0.8109)}=20 ; P=0.4296\right)$. (D) Representative images of FM4-64 dye influx before and after injury at baseline and after addition of PM subject serum. (E) Sarcolemmal resealing kinetics depicted in $\mathbf{D}$. (F) AUC calculations of $\mathbf{E}$ representing total dye influx over time $\left(n=3\right.$ and 8 for Basal and Serum, respectively; $t$ test, $\left.t_{(\mathrm{df}=4.751)}=9 ; P=0.001\right)$. (G) Representative images of FM4-64 dye influx before and after injury at baseline and after addition of DM subject serum. (H) Sarcolemmal resealing kinetics depicted in G. (I) AUC calculations of $\mathbf{H}$ ( $n=6$ and 5 for Basal and Serum, respectively; $t$ test, $t_{(\mathrm{df}=4.717)}=4.8 ; P=0.0057$ ). Scale bars: $20 \mu \mathrm{m}$.

it is not sufficient to induce severe inflammation or degeneration of skeletal muscle (12). Foxp $3^{-/ Y}$ mice heterozygous for mutant Syt7 develop little or no skeletal muscle inflammation at 2 or 4 weeks of age. When a Foxp $3^{-/ Y}$ mutation is combined with a homozygous mutation of the Syt7 gene, robust skeletal muscle inflammation is observed (12), indicating that antigen presentation of intramuscular proteins is necessary to drive IIM progression. Adoptive transfer of lymph node cells isolated from this double-mutant mouse to Rag1-/- mice induces robust myositis, with inflammatory infiltrates consisting mainly of $\mathrm{CD}^{+}$and to a lesser extent of $\mathrm{CD}^{+} \mathrm{T}$ cells and macrophages (12). The recipient $\mathrm{Rag1}^{-/-}$mice do not show compromised membrane repair, indicating that priming of lymphocytes with abnormal antigens occurred in the $\mathrm{Foxp}^{-/ Y} S y t 7^{--}$donor mice and is sufficient to induce inflammation in the absence of the $S y t 7$ mutation.

In the present study, we expanded our previously published histological examination (12) to include both proximal (quadri- ceps) and distal (EDL, soleus) skeletal muscle from our adoptive transfer model of IIM (Figure 1 and Supplemental Figure 1). We observed significant inflammation in the quadriceps as previously reported (12), while examination of the proximal EDL and soleus muscles revealed no obvious pathology. This finding is significant because our laser injury assays were performed on distal FDB muscles isolated from the foot with no overt signs of inflammation present at the time of laser injury. Our animal model not only recapitulates the pattern of inflammation observed in IIM, but also reveals a possible mechanism sustaining the autoimmune response that targets skeletal muscle.

Evaluation of sarcolemmal integrity by IgG staining demonstrated higher levels of $\mathrm{IgG}^{+}$muscle fibers in the quadriceps (Figure 2) accompanied by areas of severe inflammation and fatty tissue replacement (Figure 1A). Interestingly, the levels of $\mathrm{IgG}^{+}$ fibers observed in EDL and soleus muscles (Figure 2, B and D, 
A
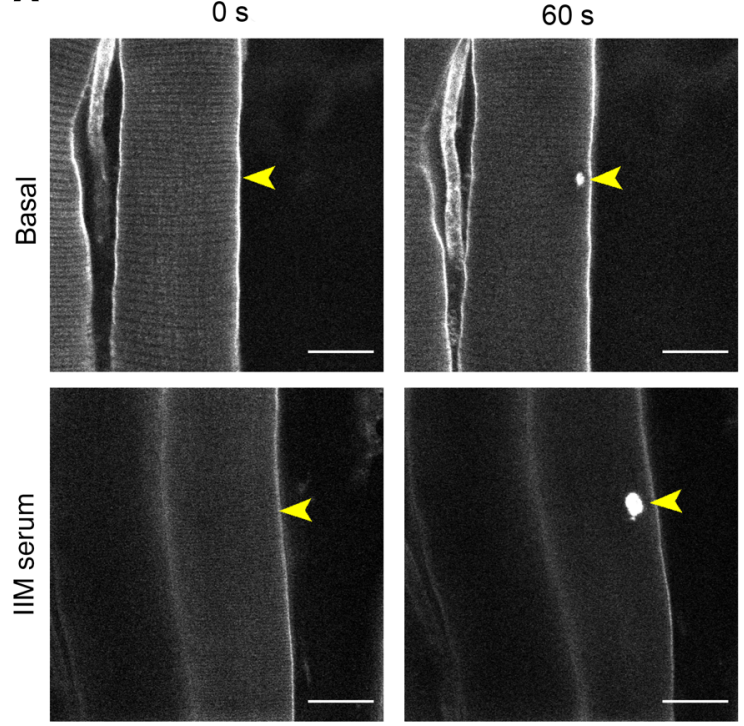

B

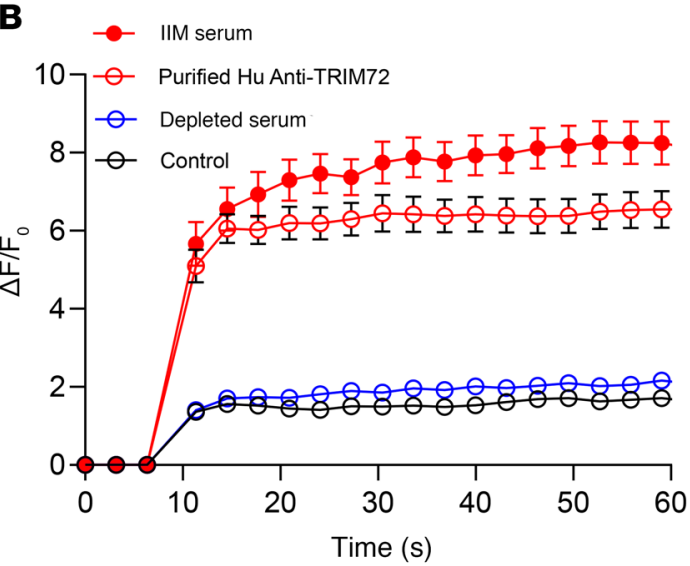

$0 \mathrm{~s}$
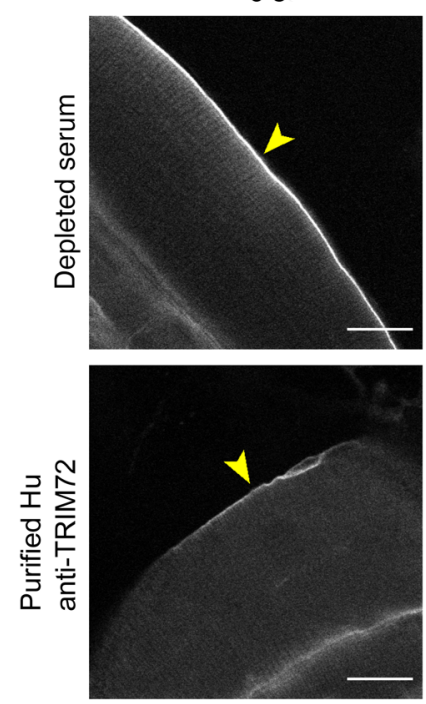

$60 \mathrm{~s}$
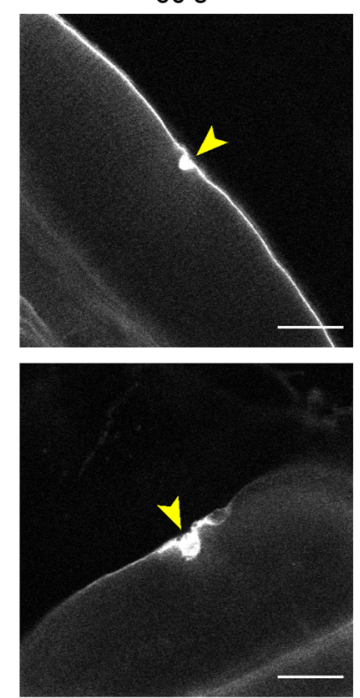

C

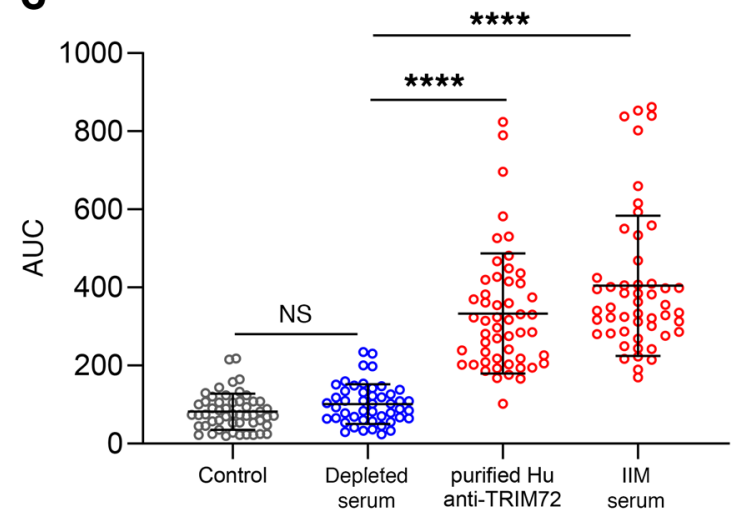

Figure 8. Depletion of TRIM72 autoantibodies from IIM patient sera rescues the normal sarcolemma resealing phenotype in healthy skeletal muscle. Yellow arrowheads indicate sites of injury. (A) Representative images of FM4-64 dye influx before (0 seconds) and after (60 seconds) injury at baseline (Basal) or after addition of IIM patient sera, IIM patient sera depleted of TRIM72 autoantibodies, and exogenous delivery of purified Hu anti-TRIM72 recovered from IIM patient sera. Scale bars: $20 \mu \mathrm{m}$. (B) Sarcolemmal resealing kinetics measured every 3 seconds for 60 seconds as depicted in $\mathbf{A}$. Data are represented as mean \pm SEM. (C) AUC calculations of B. ( $n=52,48,52$, and 51 for Basal, Depleted serum, IIM serum, and Purified Hu anti-TRIM72, respectively. ANOVA, $F_{(3.20)}=48.16, P<0.0001$; Tukey's test: Basal vs. Depleted serum, $P=0.95$; Depleted serum vs. Purified Hu anti-TRIM72, $P<0.0001$; Depleted serum vs. IIM serum, $P<0.0001$.) Data are representative of 3 IIM patient serum samples depleted of TRIM72 autoantibodies. Data are represented as the mean \pm SD.

and Supplemental Figure 3) were significantly elevated even though no inflammatory infiltrates or fatty tissue replacement were detected by histology (Figure 1B and Supplemental Figure 1). The observed reduction in sarcolemmal integrity in distal skeletal muscle is not the result of cytotoxic immune cell activity as determined by histological analysis. The decrease in sarcolemmal integrity in the absence of inflammation is an intriguing finding, and the current study was designed to evaluate a mechanistic explanation of this observation.

Histological and immunohistochemical findings show that decreased sarcolemmal integrity in distal skeletal muscle is a consequence of IIM occurring in the absence of inflammation. Our findings suggest that a defect in sarcolemmal resealing may be an early event that precedes the skeletal muscle degeneration and inflammation associated with IIM. To establish whether a lack of Tregs alone is sufficient to decrease sarcolemmal resealing capac- ity, laser injury assays were performed on $\mathrm{Foxp}^{-/ Y}$ mice lacking mature Tregs. We observed no difference in sarcolemmal resealing kinetics in Treg-deficient mice versus C57BL controls (Figure 3). We did observe a significant reduction in resealing capacity of Rag1 ${ }^{-/}$mice receiving adoptive transfer of lymph node cells from the Foxp $3^{-/ Y}$ Syt $7^{-/}$double-mutant background versus Rag1 $1^{-/}$mice receiving sham adoptive transfer (Figure 4). The observed defect in sarcolemmal resealing was significantly more pronounced at 1 week after adoptive transfer versus 4 weeks, with both time points significantly different from sham mice (Figure 4C). A possible explanation for the time-dependent magnitude of dye influx is the serum half-life of mouse IgG (6-8 days; refs. 51-53) and the low number of adoptively transferred B cells that persist at 4 weeks after adoptive transfer (12). Without a renewable B cell population in Rag1 $^{-/}$mice, autoantibody production is limited. The laser injury assays used in this study allow us to qualitatively eliminate com- 
plement-induced membrane attack complex (MAC) formation; the lipophilic dye FM4-64 used in this assay would flood muscle fibers should MAC be present on the sarcolemma. We did not observe muscle fibers become saturated with dye over the time course of our laser injury experiments.

Autoantibody production is a hallmark of IIM, and we hypothesized that proteins involved in the sarcolemmal resealing process represented a pool of antigens in the Treg-deficient/dysfunctional milieu of IIM. Autoantibodies against sarcolemmal repair proteins represent a potential mechanism that drives the progression of IIM by binding proteins critical to the resealing process and thereby preventing repair patch formation. Binding of antibodies to proteins involved in the recruitment and nucleation of repair patch vesicles could result in a "leaky" muscle fiber following an injury that leads to muscle degeneration and necrosis. We developed an ELISA to detect autoantibodies against TRIM72, a critical protein component in sarcolemmal resealing $(37,38,41,42,57)$, and found significantly elevated levels of TRIM72 autoantibodies in the Foxp $3^{-/ Y}$ Syt7-- mice (Figure 5A) and TRIM72 autoantibodies at 1 week after adoptive transfer in Rag1 $^{-/-}$mice (Figure 5B). TRIM72 autoantibody levels did not reach statistical significance in $\mathrm{Ragl}^{-1-}$ mice at 4 weeks after adoptive transfer because of the degree of within-group variance; however, a number of these mice had levels well above those observed at 1 week after adoptive transfer (Figure 5B). One interpretation of this finding is that a relatively low titer of antibodies against TRIM72 is sufficient to induce a sarcolemmal resealing defect, which is supported by our finding that IIM subject sera with low levels of anti-TRIM72 (defined as $<1$ SD observed in healthy subjects) significantly reduce membrane resealing capacity (Supplemental Figure 12A). Additionally, unidentified autoantibodies targeting proteins involved in sarcolemmal resealing or stability may be present in our adoptive transfer model of myositis and IIM subject sera. ELISA findings of elevated TRIM72 autoantibody levels in IIM, together with our data demonstrating that sarcolemmal resealing capacity is significantly reduced, are suggestive that autoantibodies targeting proteins involved in the sarcolemmal resealing response are detrimentally affecting distal skeletal muscles.

We also screened for TRIM72 autoantibodies in 103 IIM subject serum samples alongside serum samples from healthy control subjects. $11.5 \%$ of DM sera and $11.8 \%$ of PM sera tested had high levels of anti-TRIM72 (defined as $>2$ SD of healthy sera; Figure $5 \mathrm{C})$. Using a less stringent cutoff of $>1$ SD of healthy sera, $34.6 \%$ and $21.6 \%$ of DM and PM subject sera, respectively, had elevated levels of anti-TRIM72. Our observation of sarcolemmal resealing defects in the adoptive transfer mouse model at 1 and 4 weeks after adoptive transfer indicates that in a Treg-deficient/dysfunctional milieu, a slight increase in anti-TRIM72 levels correlates with reduced sarcolemmal resealing capacity (Figures 4 and 7). Our finding that $11.5 \%$ of DM sera and $11.8 \%$ of PM sera have high levels of anti-TRIM72 is also of significance. The percentage of IIM subjects with elevated TRIM72 autoantibodies is similar to those observed for established myositis-specific and myositisassociated autoantibodies. Autoantibodies against the most thoroughly investigated autoantigen, histidyl transfer RNA synthetase (Jo-1), are routinely found at a prevalence of $15 \%-30 \%$ in subjects diagnosed with PM and DM $(58,59)$. Circulating TRIM72 protein has been observed in healthy human sera $(60,61)$ at low levels $(143.80 \pm 78.1 \mathrm{pg} / \mathrm{mL}$; ref. 62$)$, demonstrating that a potentially autoantigenic pool of TRIM72 protein is readily available in a Treg-deficient milieu. The levels of TRIM72 protein in serum from our adoptive transfer model of IIM were below the levels that could be detected by Western blotting (Supplemental Figure 14). Future studies on the prevalence of TRIM72 antibodies in larger cohorts of IIM patients in which longitudinal measurements can be made will be necessary to resolve the extent to which TRIM72 antibodies can be correlated with the progression of myositis.

Based on our observations of decreased sarcolemmal integrity in the absence of inflammation, together with our ELISA data demonstrating elevated levels of TRIM72 autoantibodies in our adoptive transfer model of IIM and IIM subject sera, we sought to determine whether antibodies against TRIM72 are sufficient to reduce sarcolemmal resealing capacity in healthy skeletal muscle. Exogenous delivery of a polyclonal antibody against TRIM72 was sufficient to significantly reduce sarcolemmal resealing capacity in FDB muscles from healthy C57BL mice (Figure 6). To establish that IIM subject sera with elevated anti-TRIM72 levels compromise sarcolemmal resealing, we repeated our laser injury assays using exogenous delivery of IIM subject sera determined to have elevated anti-TRIM72. We observed a significant decrease in sarcolemmal resealing capacity regardless of IIM subtype versus healthy subjects (Figure 7). To determine a direct causal effect of TRIM72 autoantibodies resulting in the observed decrease in sarcolemmal resealing, IIM subject sera with high anti-TRIM72 levels were depleted of autoantibodies. Sarcolemmal resealing of healthy skeletal muscle in the presence of subject sera depleted of TRIM72 autoantibodies rescued a normal sarcolemmal resealing phenotype, while the addition of the isolated and purified TRIM72 autoantibodies from subject serum resulted in decreased resealing capacity (Figure 8 and Supplemental Figure 15).

Decreased resealing capacity was observed at both high and low anti-TRIM72 levels (Figure 6 and Supplemental Figure 12A). Additionally, 1 of the 3 IIM subject serum samples depleted of TRIM72 autoantibodies only partially rescued a normal sarcolemmal resealing phenotype in healthy skeletal muscle (Supplemental Figure 15C). Given the heterogeneity of IIM autoantibody profiles observed in humans, it is unlikely that the antibody-driven resealing defect we observed is exclusively the result of autoantibodies targeting one specific membrane resealing/repair protein. Several proteins are known to be involved in the sarcolemmal resealing and repair response, including but not limited to TRIM72 (37-39), SYT7 $(10,13,17,48-50)$, calpain $(40,63,64)$, annexins $(65,66)$, and dysferlin $(28,29)$. In the present study, we only evaluated the role of anti-TRIM72 in sarcolemmal resealing defects. Previous studies of the functional role of the synaptotagmin family of proteins in membrane resealing and repair have demonstrated that polyclonal antibodies targeting synaptotagmin proteins can reduce membrane resealing $\left(17,48^{-50}\right)$. We show that IIM subject sera determined to have low levels of TRIM72 autoantibodies have a detrimental effect on sarcolemmal resealing (Supplemental Figure 12A). IIM subject serum with low anti-TRIM72 levels negatively affecting sarcolemmal resealing suggests that other factors potentially contribute to this phenotype. This raises the possibility that unidentified autoantibodies are targeting additional sarcolemmal repair proteins. 
Recently, autoantibodies determined to target the musclespecific intermediate filament desmin were identified in inclusion body myositis (67). Desmin has been identified as an autoantigen in autoimmune conditions other than $\operatorname{IIM}(68,69)$, and, while not directly involved in sarcolemmal repair, disruption of the cytoskeletal architecture of muscle fibers could destabilize the sarcolemma and produce the decreased membrane integrity observed by IgG staining (Figure 2 and Supplemental Figure 3), further supporting our hypothesis that autoantibodies disrupt sarcolemmal integrity.

We propose a mechanism through which autoantibodies that target critical components of sarcolemmal resealing and repair lead to the progression of pathophysiology observed in IIM. A deficiency and/or dysfunction of Tregs is associated with IIM, which could facilitate the priming of the autoimmune response. The catalyst for this Treg dysfunction remains unclear; however, environmental and genetic factors have been proposed. Several scenarios may lead to exposure of intramuscular antigens to the Tregdeficient milieu, including trauma to skeletal muscle, viral or bacterial infection, or the additive effect of sarcolemmal disruptions and repair due to normal physical exertion over time. Exposure of skeletal muscle antigens, particularly those involved in sarcolemmal resealing and repair, should be among the first and most abundant antigens presented to naive and/or autoreactive immune cells, resulting in autoantibody production and inflammation.

The exact mechanism of sarcolemmal resealing and repair has yet to be fully elucidated, and several models of plasma membrane repair have been proposed (reviewed by Blazek et al., ref. 18). A common feature of the proposed models is the exposure of intracellular proteins to the extracellular space in the form of vesicle exocytosis and patch formation to seal disruptions, membrane budding and blebbing, or shedding of the injured portion of the membrane during the repair/remodeling response. The exposure of sarcolemmal resealing and repair proteins could not only stimulate autoantibody production but also provide a substrate for antibodies and antigen-presenting cells. Our observation that distal skeletal muscle has defective sarcolemmal resealing in the absence of inflammation supports the hypothesis that antigen presentation due to normal physiological stress on skeletal muscle provides constant stimulation to antibody-producing plasma cells in a Treg-deficient milieu. When a secondary insult occurs to skeletal muscle, the autoimmune response is primed to initiate a significant inflammatory response at the site of trauma. Our findings represent a mechanism that contributes to the progression of IIM when decreased sarcolemmal integrity induces a positive-feedback loop of decreased sarcolemmal resealing and antigen presentation.

\section{Methods}

Additional details on methods can be found in Supplemental Methods.

Myositis serum samples and skeletal muscle biopsies. Human serum samples were acquired from the University of Pittsburgh Myositis Center and The Ohio State University Wexner Medical Center, Division of Rheumatology and Immunology. Myositis patient skeletal muscle biopsies were a gift from Zarife Sahenk (Nationwide Children's Hospital, Columbus, Ohio, USA).

TRIM72 polyclonal antibody. The custom affinity-purified TRIM72 polyclonal antibody used in injury assays and Western blots has previously been described (70) and was generated in New Zea- land white rabbits immunized with full-length recombinant human TRIM72 (rhTRIM72) protein by Pacific Immunology Group. Antibody titer as determined by ELISA is 1:500,000. See Supplemental Figure 13 for antibody specificity.

Mouse breeding. Male C57BL/6 (B6) mice, female B6.Cg-FoxP3 ${ }^{\mathrm{s} / \mathrm{x}}$ / $\mathrm{J}\left(\mathrm{FoxP}^{+/-}\right)$mice, and female B6.129S7-Rag1 ${ }^{\mathrm{tm} 1 \mathrm{Mom}} / \mathrm{J}$ (Rag1/-) mice were obtained from The Jackson Laboratory. Female B6.129S1Syt $7^{\mathrm{tm} 1 \mathrm{Nan}} / \mathrm{J}$ (Syt $\left.\mathrm{VII}^{+/-}\right)$mice were maintained at The Ohio State University and were originally a gift from Norma Andrews (University of Maryland, College Park, Maryland, USA). Foxp $3^{-/ Y}$ Syt $7^{-/-}$mice were generated as previously described (12). Briefly, female B6.129S1Syt $7^{\mathrm{tm} 1 \mathrm{Nan}} / \mathrm{J}\left(S y t 7^{+/-}\right)$mice were crossbred with male C57BL/6 mice to generate male $S y t 7^{+/-}$mice. The $S y t 7^{+/-}$males were then mated to $S y t 7^{+/-}$females to produce $S y t 7^{-/-}$progeny. B6/Cg-Foxp $3^{\text {sf } / x} / \mathrm{J}\left(F o x p 3^{+/-}\right)$ females were mated to $S y t 7^{-/-}$male mice to produce Foxp $3^{+/-} S y t 7^{-1-}$ female progeny. The Foxp $3^{-/ Y}$ Syt $7^{-/-}$mice used in all experiments were then generated by mating of $\mathrm{Foxp}^{+/-} \mathrm{Syt7^{-/- }}$ female mice with Syt $7^{-/-}$males.

Adoptive transfer. Foxp $3^{-/ Y}$ Syt7-1- double-mutant mice were euthanized at 25 days of age by $\mathrm{CO}_{2}$ inhalation followed by cervical dislocation. Whole blood was collected by cardiac puncture, and serum was isolated for analysis. Lymph nodes were collected from each mouse (tonsillar, submandibular, axillary, and inguinal) and placed into sterile PBS. Pooled lymph nodes were mechanically sheared to release lymphocytes using the plunger of a $10-\mathrm{mL}$ syringe and $100-\mu \mathrm{m}$ cell strainer. A single-cell suspension consisting of $1.5 \times 10^{7}$ cells in 200 $\mu \mathrm{L}$ sterile PBS was injected intramuscularly into hamstring muscles of male $R a g 1^{1 /-}$ mice. Recipient mice were monitored every other day for disease progression and euthanized at 1 and 4 weeks or loss of $20 \%$ body mass. Serum and individual proximal (quadriceps, hamstring, and gluteus) and distal (tibialis anterior, gastrocnemius, soleus, EDL, and flexor digitorum brevis) muscles were collected.

Histology. Skeletal muscles from the mouse hind limbs were dissected and fixed for 72 hours at room temperature in $10 \%$ neutral-buffered formalin. Tissue was processed and paraffinembedded according to standard protocols, cut into $5-\mu \mathrm{m}$ sections, and used for $\mathrm{H} \& \mathrm{E}$ and immunohistochemical staining.

IgG staining and analysis. Five-micrometer paraffin-embedded sections of quadriceps, EDL, and soleus muscles underwent epitope retrieval using citrate buffer. Sections were then blocked in 2\% BSA for 1 hour and incubated overnight with goat anti-mouse IgG antibody conjugated with Alexa Fluor 488 (Invitrogen, catalog A11001). Analysis of the percentage area $\operatorname{IgG}^{+}$was conducted using ImageJ (NIH). The area of an image representing skeletal muscle was quantified by visual setting of a threshold that included the entirety of the muscle section to be analyzed and exclusion of histological artifacts and dark background. A second threshold corresponding to areas of each skeletal muscle section positive for IgG was applied to each image. The percentage area of $\mathrm{IgG}^{+}$skeletal muscle was then determined by division of the $\operatorname{IgG}^{+}$area by the total area of each image representing skeletal muscle.

ELISA. Ninety-six-well ELISA plates were coated with $50 \mathrm{ng}$ rhTRIM72 in bicarbonate/carbonate coating buffer overnight at $4^{\circ} \mathrm{C}$. The remaining protein binding sites were blocked with $2 \%$ BSA in coating buffer for 2 hours at room temperature. Serum samples from indicated mice or IIM subject samples diluted 1:50 in 1\% BSA were then added to each well and incubated for 2 hours at room tempera- 
ture. After the plate was washed 3 times with PBS-Tween-20, antihuman IgG-HRP (Abcam, catalog ab6858) or anti-mouse IgG-HRP antibody (Abcam, catalog ab205719) diluted 1:40,000 in 1\% BSA was added to each well and incubated for 2 hours at room temperature. After washing, $100 \mu \mathrm{L}$ TMB ELISA substrate was added to each well and incubated for 20 minutes. OD values were read at $370 \mathrm{~nm}$ according to the manufacturer's instructions.

Laser injury assay. Intact FDB muscles were dissected from indicated mice and affixed to a $35-\mathrm{mm}$ glass-bottom imaging dish with commercially available liquid bandage. Three hundred microliters Tyrode's solution supplemented with $2 \mathrm{mM} \mathrm{Ca}^{2+}$ and $10.5 \mu \mathrm{M} \mathrm{FM} 4-$ 64 dye was added to the glass-bottom microwell of each dish to completely cover FDB muscles. For laser injury assays using IIM subject serum, either healthy or IIM sera were added to Tyrode's solution at a dilution of 1:200. Laser injury assays used purified anti-TRIM72 antibody dilutions as indicated. Assays were conducted using a FluoView FV1000 MPE multiphoton confocal laser scanning microscope equipped with a DeepSee MaiTai titanium-sapphire laser and $\times 251.05$ NA water immersion objective. A $157-\mathrm{nm}^{2}$ region of interest was selected on the sarcolemma of individual FDB muscle fibers as the injury site. Images were acquired approximately every 3 seconds for 20 frames: 3 frames before injury, followed by a 5-second infrared laser irradiation at $23 \%-40 \%$ maximal power, and 17 frames after injury. For each comparison, the infrared laser power was held constant for control and experimental groups. Membrane damage was quantified by measurement of fluorescence intensity at the site of injury for each frame. Background fluorescence was measured using a region of interest of the same area immediately adjacent to the injury site, allowing for bleaching correction. Membrane resealing kinetics is represented as a curve corresponding to the change in fluorescence intensity over time $\left(\Delta F / F_{0}\right)$ calculated for each time point measured. Total dye uptake after injury was quantified by calculation of the area under the curve (AUC) representing the mean membrane resealing kinetics for each experimental group.

Rotation damage assay. HEK293T/17 cells $\left(1 \times 10^{5}\right.$; ATCC, CRL11268) were seeded in 2-mL flat-bottom microcentrifuge tubes in 500 $\mu \mathrm{L}$ of DMEM complete medium and allowed to adhere overnight. The medium was aspirated from each tube, cells were washed 3 times with PBS, and $200 \mu \mathrm{L}$ of Tyrode's buffer supplemented with $2 \mathrm{mM} \mathrm{Ca}^{2+}$ was added to each tube. Twenty microliters $(7.23 \mathrm{mg} \pm 0.63 \mathrm{mg})$ of $\leq 106-\mu \mathrm{m}$ glass beads were added by pipetting directly from the dry stock. Tubes were then placed in a hematological mixer and rotated end over end for 15 rotations. Following rotation, tubes were removed from the mixer and $10 \mu \mathrm{L}$ of supernatant transferred to a 96-well plate. $\mathrm{LDH}$ release was measured to determine membrane resealing capacity.

TRIM72 autoantibody depletion. Recombinant human TRIM72 protein was covalently immobilized to NHS-activated magnetic beads (Pierce Biotechnology, catalog 88826) according to the manufacturer's instructions. One hundred microliters of rhTRIM72-conjugated bead slurry was added to a $1.5-\mathrm{mL}$ centrifuge tube and placed in a magnetic stand, and the supernatant was discarded. Three hundred microliters of IIM sera was added to each tube and incubated with end-over-end rotation for 2 hours. Beads were then collected with a magnetic stand and autoantibody-depleted serum collected and reserved for downstream assays. Autoantibodies bound to rhTRIM72-conjugated magnetic beads were then eluted with $300 \mu \mathrm{L}$ of $0.1 \mathrm{M}$ glycine buffer ( $\mathrm{pH}$ 2.0), and low $\mathrm{pH}$ was neutralized with $1 \mathrm{M}$ phosphate buffer ( $\mathrm{pH}$ 7.4). Each serum sample underwent 3 rounds of autoantibody depletion, and eluted antibodies were then pooled and used for downstream assays.

Statistics. Graphical representation and statistical analysis of data were performed using GraphPad Prism version 8.1. Data were analyzed by a 2 -tailed $t$ test assuming unequal variances, Welch's $t$ test for unbalanced designs, or ANOVA as appropriate. ANOVA post hoc analysis used Tukey's honestly significant difference test or Dunnett's test for comparisons. A P value of less than 0.05 was considered significant.

Study approval. All mice were housed at The Ohio State University Wexner Medical Center (OSUWMC) vivarium under veterinarian supervision in a biosafety level 3 barrier facility. All animal protocols were approved by the Institutional Animal Care and Use Committee at OSUWMC.

The IIM subject sera used were not specifically collected for this study. Individual serum samples were received deidentified with a 9-digit identification number, and the investigators did not have access to identifiable information. Therefore, the experiments used in this study were not considered human-subjects research.

\section{Author contributions}

KEM, NW, and WNJ conceived and designed the research studies. Writing of the manuscript and preparation of figures were performed by KEM. Critical evaluation of the manuscript was provided by NW, WNJ, RA, and CVO. KEM, NY, JH, and KJ performed the adoptive transfer experiments. Mouse skeletal muscle dissection and mouse serum collection were performed by EXB. Histology and immunohistochemistry were performed by KEM, BJP, and TAK. Rotational damage assays were performed by TAK and AC. Laser injury assays were conducted and analyzed by KEM, BJP, and AC. Western blotting was performed by KEM, TG, and MALP. ELISA development and assays were performed by KEM. RA and CVO provided serum and muscle biopsy samples. Autoantibody depletion and purification were performed by KEM.

\section{Acknowledgments}

The research reported in this publication was partially supported by a National Institute of Arthritis and Musculoskeletal and Skin Diseases, NIH, National Research Service Award individual predoctoral fellowship under award number 1F31AR071745-01 to KEM. Images were generated at The Ohio State University Campus Microscopy and Imaging Facility, which is supported in part by grant P30 CA016058, National Cancer Institute, Bethesda, Maryland, USA. The content is solely the responsibility of the authors and does not necessarily represent the official views of the NIH. The authors thank the participants and staff involved in these studies. The authors also acknowledge the contributions of Zarife Sahenk (Nationwide Children's Research Institute, Columbus, Ohio, USA), Lisa G. Rider, and Frederick W. Miller (NIH/National Institute of Environmental Health Sciences, Bethesda, Maryland, USA).

Address correspondence to: Noah Weisleder, Department of Physiology and Cell Biology, Dorothy M. Davis Heart and Lung Research Institute, The Ohio State University Wexner Medical Center, 473 W. 12th Avenue, Columbus, Ohio 43210-1252, USA. Phone: 614.292.5321; Email: Noah.Weisleder@osumc.edu. 
1. Betteridge Z, McHugh N. Myositis-specific autoantibodies: an important tool to support diagnosis of myositis. J Intern Med. 2016;280(1):8-23.

2. Haq SA, Tournadre A. Idiopathic inflammatory myopathies: from immunopathogenesis to new therapeutic targets. Int J Rheum Dis. 2015;18(8):818-825.

3. Figarella-Branger D, Civatte M, Bartoli C, Pellissier JF. Cytokines, chemokines, and cell adhesion molecules in inflammatory myopathies. Muscle Nerve. 2003;28(6):659-682.

4. Christopher-Stine L, Plotz PH. Myositis: an update on pathogenesis. Curr Opin Rheumatol. 2004;16(6):700-706.

5. Mendez EP, et al. US incidence of juvenile dermatomyositis, 1995-1998: results from the National Institute of Arthritis and Musculoskeletal and Skin Diseases Registry. Arthritis Rheum. 2003;49(3):300-305.

6. Findlay AR, Goyal NA, Mozaffar T. An overview of polymyositis and dermatomyositis. Muscle Nerve. 2015;51(5):638-656.

7. Miller FW, et al. Genome-wide association study identifies HLA 8.1 ancestral haplotype alleles as major genetic risk factors for myositis phenotypes. Genes Immun. 2015;16(7):470-480.

8. Habers GEA, et al. Brief Report: Association of myositis autoantibodies, clinical features, and environmental exposures at illness onset with disease course in juvenile myositis. Arthritis Rheumatol. 2016;68(3):761-768.

9. Sunderkötter C, et al. Guidelines on dermatomyositis-excerpt from the interdisciplinary S2k guidelines on myositis syndromes by the German Society of Neurology. J Dtsch Dermatol Ges. 2016;14(3):321-338.

10. Chakrabarti S, et al. Impaired membrane resealing and autoimmune myositis in synaptotagmin VII-deficient mice. JCell Biol. 2003;162(4):543-549.

11. Sharma R, Zheng L, Guo X, Fu SM, Ju ST, Jarjour WN. Novel animal models for Sjögren's syndrome: expression and transfer of salivary gland dysfunction from regulatory $\mathrm{T}$ cell-deficient mice. J Autoimmun. 2006;27(4):289-296.

12. Young NA, Sharma R, Friedman AK, Kaffenberger $\mathrm{BH}$, Bolon B, Jarjour WN. Aberrant muscle antigen exposure in mice is sufficient to cause myositis in a Treg cell-deficient milieu. Arthritis Rheum. 2013;65(12):3259-3270.

13. Martens S, Kozlov MM, McMahon HT. How synaptotagmin promotes membrane fusion. Science. 2007;316(5828):1205-1208.

14. Detrait ER, Yoo S, Eddleman CS, Fukuda M, Bittner GD, Fishman HM. Plasmalemmal repair of severed neurites of PC12 cells requires $\mathrm{Ca}(2+)$ and synaptotagmin. J Neurosci Res. 2000;62(4):566-573.

15. Andrews NW. Membrane resealing: synaptotagmin VII keeps running the show. Sci STKE. 2005;2005(282):pe19.

16. Roy D, et al. A process for controlling intracellular bacterial infections induced by membrane injury. Science. 2004;304(5676):1515-1518.

17. Chakrabarti S, et al. Impaired membrane resealing and autoimmune myositis in synaptotagmin VII-deficient mice. J Cell Biol. 2003;162(4):543-549.

18. Blazek AD, Paleo BJ, Weisleder N. Plasma mem- brane repair: a central process for maintaining cellular homeostasis. Physiology (Bethesda). 2015;30(6):438-448.

19. McNeil PL, Ito S. Gastrointestinal cell plasma membrane wounding and resealing in vivo. Gastroenterology. 1989;96(5 pt 1):1238-1248.

20. McNeil PL, Vogel SS, Miyake K, Terasaki M. Patching plasma membrane disruptions with cytoplasmic membrane. J Cell Sci. 2000;113 (pt 11):1891-1902.

21. McNeil PL, Kirchhausen T. An emergency response team for membrane repair. Nat Rev Mol Cell Biol. 2005;6(6):499-505.

22. McNeil PL, Miyake K, Vogel SS. The endomembrane requirement for cell surface repair. Proc Natl Acad Sci U S A. 2003;100(8):4592-4597.

23. McNeil PL, Steinhardt RA. Plasma membrane disruption: repair, prevention, adaptation. Annu Rev Cell Dev Biol. 2003;19:697-731.

24. Togo T, Krasieva TB, Steinhardt RA. A decrease in membrane tension precedes successful cell-membrane repair. Mol Biol Cell. 2000;11(12):4339-4346.

25. Steinhardt RA. The mechanisms of cell membrane repair: a tutorial guide to key experiments. Ann N Y Acad Sci. 2005;1066:152-165.

26. Miyake K, McNeil PL. Vesicle accumulation and exocytosis at sites of plasma membrane disruption. J Cell Biol. 1995;131(6 pt 2):1737-1745.

27. Steinhardt RA, Bi G, Alderton JM. Cell membrane resealing by a vesicular mechanism similar to neurotransmitter release. Science. 1994;263(5145):390-393.

28. Doherty KR, McNally EM. Repairing the tears: dysferlin in muscle membrane repair. Trends Mol Med. 2003;9(8):327-330.

29. Bansal D, et al. Defective membrane repair in dysferlin-deficient muscular dystrophy. Nature. 2003;423(6936):168-172.

30. Bazan NG, Marcheselli VL, Cole-Edwards K. Brain response to injury and neurodegeneration: endogenous neuroprotective signaling. Ann N Y Acad Sci. 2005;1053:137-147.

31. Casciola-Rosen L, Mammen AL. Myositis autoantibodies. Curr Opin Rheumatol. 2012;24(6):602-608.

32. Casciola-Rosen L, et al. Enhanced autoantigen expression in regenerating muscle cells in idiopathic inflammatory myopathy. J Exp Med. 2005;201(4):591-601.

33. Espinosa A, et al. The Sjogren's syndromeassociated autoantigen Ro52 is an E3 ligase that regulates proliferation and cell death. J Immunol. 2006;176(10):6277-6285.

34. Kawai T, Akira S. Regulation of innate immune signalling pathways by the tripartite motif (TRIM) family proteins. EMBO Mol Med 2011;3(9):513-527.

35. Ozato K, Shin DM, Chang TH, Morse HC. TRIM family proteins and their emerging roles in innate immunity. Nat Rev Immunol. 2008;8(11):849-860.

36. Nagre $\mathrm{N}$, et al. Inhibition of macrophage complement receptor CRIg by TRIM72 polarizes innate immunity of the lung. Am J Respir Cell Mol Biol. 2018;58(6):756-766.

37. Cai C, et al. MG53 nucleates assembly of cell membrane repair machinery. Nat Cell Biol. 2009;11(1):56-64.
38. Weisleder $\mathrm{N}$, et al. Recombinant MG53 protein modulates therapeutic cell membrane repair in treatment of muscular dystrophy. Sci Transl Med. 2012;4(139):139ra85.

39. Alloush J, Weisleder N. TRIM proteins in therapeutic membrane repair of muscular dystrophy. JAMA Neurol. 2013;70(7):928-931.

40. Mellgren RL, Zhang W, Miyake K, McNeil PL. Calpain is required for the rapid, calciumdependent repair of wounded plasma membrane. J Biol Chem. 2007;282(4):2567-2575.

41. He B, et al. Enhancing muscle membrane repair by gene delivery of MG53 ameliorates muscular dystrophy and heart failure in $\delta$-Sarcoglycan-deficient hamsters. Mol Ther. 2012;20(4):727-735.

42. Cao CM, et al. MG53 constitutes a primary determinant of cardiac ischemic preconditioning. Circulation. 2010;121(23):2565-2574.

43. Wang X, et al. Cardioprotection of ischemia/ reperfusion injury by cholesterol-dependent MG53-mediated membrane repair. Circ Res. 2010;107(1):76-83.

44. Zhang Y, et al. MG53 participates in ischaemic postconditioning through the RISK signalling pathway. Cardiovasc Res. 2011;91(1):108-115.

45. Fontenot JD, Gavin MA, Rudensky AY. Pillars Article: Foxp3 programs the development and function of CD4+CD25+ regulatory T cells. Nat. Immunol. 2003. 4:330-336. JImmunol. 2017;198(3):986-992.

46. Hadaschik EN, et al. Regulatory T cell-deficient scurfy mice develop systemic autoimmune features resembling lupus-like disease. Arthritis Res Ther. 2015;17:35.

47. Sharma R, Jarjour WN, Zheng L, Gaskin F, Fu SM, Ju ST. Large functional repertoire of regulatory T-cell suppressible autoimmune T cells in scurfy mice. J Autoimmun. 2007;29(1):10-19.

48. Elferink LA, Peterson MR, Scheller RH. A role for synaptotagmin (p65) in regulated exocytosis. Cell. 1993;72(1):153-159.

49. Martinez I, Chakrabarti S, Hellevik T, Morehead J, Fowler K, Andrews NW. Synaptotagmin VII regulates $\mathrm{Ca}(2+)$-dependent exocytosis of lysosomes in fibroblasts. J Cell Biol. 2000;148(6):1141-1149.

50. Mikoshiba K, et al. Role of the C2A domain of synaptotagmin in transmitter release as determined by specific antibody injection into the squid giant synapse preterminal. Proc Natl Acad Sci U S A. 1995;92(23):10703-10707.

51. Vieira P, Rajewsky K. The half-lives of serum immunoglobulins in adult mice. Eur J Immunol. 1988;18(2):313-316.

52. Vieira P, Rajewsky K. The bulk of endogenously produced IgG2a is eliminated from the serum of adult C57BL/6 mice with a half-life of 6-8 days. Eur Immunol. 1986;16(7):871-874.

53. Ghetie V, Ward ES, Vitetta ES. Pharmacokinetics of antibodies and immunotoxins in mice and humans. In: Figg WD, McLeod HL, eds. Handbook of Anticancer Pharmacokinetics and Pharmacodynamics. Totowa, New Jersey, USA: Humana Press; 2004:475-498.

54. Ceribelli A, De Santis M, Isailovic N, Gershwin $\mathrm{ME}$, Selmi C. The immune response and the pathogenesis of idiopathic inflammatory myo- 
sitis: a critical review. Clin Rev Allergy Immunol. 2017;52(1):58-70.

55. Gunawardena H, Betteridge ZE, McHugh NJ. Myositis-specific autoantibodies: their clinical and pathogenic significance in disease expression. Rheumatology (Oxford). 2009;48(6):607-612.

56. Antiga E, et al. Characterization of regulatory $\mathrm{T}$ cells in patients with dermatomyositis. J Autoimmun. 2010;35(4):342-350.

57. Cao CM, et al. MG53 constitutes a primary determinant of cardiac ischemic preconditioning. Circulation. 2010;121(23):2565-2574.

58. Satoh M, Ceribelli A, Hirakata M, Chan EKL. Immunodiagnosis of autoimmune myopathies. In: Detrick B, Schmitz JL, Hamilton RG, eds. Manual of Molecular and Clinical Laboratory Immunology. 8th ed. Washington, DC, USA: ASM Press; 2016:878-887.

59. Satoh M, Tanaka S, Ceribelli A, Calise SJ, Chan EK. A comprehensive overview on myositisspecific antibodies: new and old biomarkers in idiopathic inflammatory myopathy. Clin Rev Allergy Immunol. 2017;52(1):1-19.
60. Lemckert FA, et al. Lack of MG53 in human heart precludes utility as a biomarker of myocardial injury or endogenous cardioprotective factor. Cardiovasc Res. 2016;110(2):178-187.

61. Wu HK, et al. Glucose-sensitive myokine/cardiokine MG53 regulates systemic insulin response and metabolic homeostasis. Circulation. 2019;139(7):901-914.

62. Chen Z, et al. Serum levels of TRIM72 are lower among patients with colon cancer: identification of a potential diagnostic marker. Tohoku J Exp Med. 2018;245(1):61-68.

63. Redpath GM, et al. Calpain cleavage within dysferlin exon 40a releases a synaptotagmin-like module for membrane repair. Mol Biol Cell. 2014;25(19):3037-3048.

64. Lek A, et al. Calpains, cleaved mini-dysferlinC72, and L-type channels underpin calciumdependent muscle membrane repair. J Neurosci. 2013;33(12):5085-5094.

65. Boye TL, et al. Annexin A4 and A6 induce membrane curvature and constriction during cell membrane repair. Nat Commun. 2017;8(1):1623.
66. Demonbreun AR, Quattrocelli M, Barefield DY, Allen MV, Swanson KE, McNally EM. An actin-dependent annexin complex mediates plasma membrane repair in muscle. JCell Biol. 2016;213(6):705-718.

67. Ray A, et al. Autoantibodies produced at the site of tissue damage provide evidence of humoral autoimmunity in inclusion body myositis. PLoS One. 2012;7(10):e46709.

68. Senécal JL, Rauch J. Hybridoma lupus autoantibodies can bind major cytoskeletal filaments in the absence of DNA-binding activity. Arthritis Rheum. 1988;31(7):864-875.

69. Varon D, et al. Human monoclonal antibody derived from an autoimmune thrombocytopenic purpura patient, recognizing an intermediate filament's determinant common to vimentin and desmin. Clin Immunol Immunopathol. 1990;54(3):454-468.

70. Gushchina LV, et al. Treatment with recombinant human MG53 protein increases membrane integrity in a mouse model of limb girdle muscular dystrophy 2B. Mol Ther. 2017;25(10):2360-2371. 